\title{
Comparing Dark Energy Survey and HST-CLASH observations of the galaxy cluster RXC J2248.7-4431: implications for stellar mass versus dark matter
}
A. Palmese,${ }^{1 \star}$ O. Lahav, ${ }^{1}$ M. Banerji, ${ }^{2}$ D. Gruen, ${ }^{3,4,5,6}$ S. Jouvel, ${ }^{1}$ P. Melchior, ${ }^{7}$
J. Aleksić, ${ }^{8}$ J. Annis, ${ }^{9}$ H. T. Diehl, ${ }^{9}$ W. G. Hartley, ${ }^{1}$ T. Jeltema, ${ }^{10}$ A. K. Romer, ${ }^{11}$
E. Rozo, ${ }^{12}$ E. S. Rykoff, ${ }^{3,4}$ S. Seitz, ${ }^{5,6}$ E. Suchyta, ${ }^{13}$ Y. Zhang, ${ }^{14}$ T. M. C. Abbott, ${ }^{15}$
F. B. Abdalla, ${ }^{1,16}$ S. Allam, ${ }^{9}$ A. Benoit-Lévy, ${ }^{1,17,18}$ E. Bertin, ${ }^{17,18}$ D. Brooks, ${ }^{1}$
E. Buckley-Geer, ${ }^{9}$ D. L. Burke,${ }^{3,4}$ D. Capozzi, ${ }^{19}$ A. Carnero Rosell,,${ }^{20,21}$
M. Carrasco Kind, ${ }^{22,23}$ J. Carretero, ${ }^{8,24}$ M. Crocce, ${ }^{24}$ C. E. Cunha, ${ }^{4}$
C. B. D’Andrea, ${ }^{19,25}$ L. N. da Costa, ${ }^{20,21}$ S. Desai, ${ }^{26,27}$ J. P. Dietrich, ${ }^{26,27}$ P. Doel, ${ }^{1}$
J. Estrada, ${ }^{9}$ A. E. Evrard, ${ }^{14,28}$ B. Flaugher, ${ }^{9}$ J. Frieman, ${ }^{9,29}$ D. W. Gerdes, ${ }^{14}$
D. A. Goldstein, ${ }^{30,31}$ R. A. Gruendl, ${ }^{22,23}$ G. Gutierrez, ${ }^{9}$ K. Honscheid, ${ }^{32,33}$
D. J. James, ${ }^{15}$ K. Kuehn, ${ }^{34}$ N. Kuropatkin, ${ }^{9}$ T. S. Li, ${ }^{35}$ M. Lima, ${ }^{20,36}$
M. A. G. Maia, ${ }^{20,21}$ J. L. Marshall, ${ }^{35}$ C. J. Miller, ${ }^{28,14}$ R. Miquel, ${ }^{8,37}$ B. Nord, ${ }^{9}$
R. Ogando, ${ }^{20,21}$ A. A. Plazas, ${ }^{38}$ A. Roodman, ${ }^{3,4}$ E. Sanchez, ${ }^{39}$ V. Scarpine, ${ }^{9}$
I. Sevilla-Noarbe, ${ }^{39,22}$ R. C. Smith, ${ }^{15}$ M. Soares-Santos, ${ }^{9}$ F. Sobreira,,${ }^{9,20}$
M. E. C. Swanson, ${ }^{23}$ G. Tarle, ${ }^{14}$ D. Thomas, ${ }^{19}$ D. Tucker ${ }^{9}$ and V. Vikram ${ }^{40}$
Affiliations are listed at the end of the paper

Accepted 2016 August 15. Received 2016 August 13; in original form 2016 January 22

\begin{abstract}
We derive the stellar mass fraction in the galaxy cluster RXC J2248.7-4431 observed with the Dark Energy Survey (DES) during the Science Verification period. We compare the stellar mass results from DES (five filters) with those from the Hubble Space Telescope Cluster Lensing And Supernova Survey (CLASH; 17 filters). When the cluster spectroscopic redshift is assumed, we show that stellar masses from DES can be estimated within 25 percent of CLASH values. We compute the stellar mass contribution coming from red and blue galaxies, and study the relation between stellar mass and the underlying dark matter using weak lensing studies with DES and CLASH. An analysis of the radial profiles of the DES total and stellar mass yields a stellar-to-total fraction of $f_{\star}=(6.8 \pm 1.7) \times 10^{-3}$ within a radius of $r_{200 \mathrm{c}}$ $\simeq 2 \mathrm{Mpc}$. Our analysis also includes a comparison of photometric redshifts and star/galaxy separation efficiency for both data sets. We conclude that space-based small field imaging can be used to calibrate the galaxy properties in DES for the much wider field of view. The technique developed to derive the stellar mass fraction in galaxy clusters can be applied to the $\sim 100000$ clusters that will be observed within this survey and yield important information about galaxy evolution.
\end{abstract}

Key words: surveys-galaxies: clusters: general-galaxies: evolution-galaxies: photometry. 


\section{INTRODUCTION}

In the last decade, large photometric galaxy surveys, such as Sloan Digital Sky Survey (SDSS), have provided us with a massive amount of data that have proven to be extremely useful for studies of cosmology. On the other hand, smaller area but deeper surveys like the Hubble Space Telescope (HST) based Cluster Lensing And Supernova Survey (CLASH) (Postman et al. 2012) allowed us to characterize single objects with unprecedented precision. The importance of finding synergies between these surveys relates to several aspects of observation (e.g. target selection, photometric calibration) and data analysis (photometric redshifts, physical properties of galaxies). This is particularly relevant for overlapping groundand space-based surveys: the higher quality that can be obtained from space can enable calibration and tests for the data collected by ground-based telescopes.

In this paper, we study the cluster of galaxies RXC J2248.7-4431 (hereafter RXJ2248). We make use of the synergies between DES and CLASH, and test in this way the performance of the early DES data at a catalogue level (i.e. without making use of the images for the results). Photometric redshift (photo- $z$ ) and stellar mass results from CLASH are also used as a validation set for DES stellar mass estimates.

The aim of this paper is twofold: the first goal is to compare between DES's wide area breadth and CLASH's small area precision for the cluster RXJ2248. In fact, checks using HST data had not been done before to test the DES data, although the similar optical filters and the additional UV and IR HST bands make CLASH an optimal candidate for validation and quantifying uncertainties of photometry, photo- $z \mathrm{~s}$ and stellar masses. The second is to illustrate how an analysis of the stellar mass distribution of this massive cluster over the wider Dark Energy Camera (DECam) field of view can be done.

In Section 2, we start by describing the two surveys considered. The cluster is described in Section 3. The comparison of DES and CLASH, in terms of photometric redshifts and star/galaxy separation, is presented in Section 4. Section 5 contains the second part of this paper, where we present the stellar mass results obtained from CLASH and DES, and compare the DES stellar masses to the total mass from the DES weak lensing analysis by Melchior et al. (2015). In the following, we assume a concordance $\Lambda$ cold dark matter $(\Lambda \mathrm{CDM})$ cosmological model with $\Omega_{\mathrm{m}}=0.3, \Omega_{\Lambda}=0.7$ and $h=0.7$. In this cosmology, 1 arcmin corresponds to a physical transverse length of $295 \mathrm{kpc}$ at the cluster redshift $z=0.3475$.

The notation adopted in this paper for the cluster mass and radius follows the one often used in literature. The radii of spheres around the cluster centre are written as $r_{\Delta m}$ and $r_{\Delta c}$ where $\Delta$ is the overdensity of the sphere with respect to the mean matter density (subscript $m$ ) or the critical density (subscript $c$ ) at the cluster redshift. Masses inside those spheres are therefore $M_{\Delta m}=\Delta \frac{4 \pi}{3} r_{\Delta m}^{3} \rho_{\mathrm{m}}$ and similarly for $M_{\Delta c}$. In the following, we quote $\Delta=200$, which is the density contrast at virialization for a dark matter halo.

\section{DATA}

The data used for the analyses developed for this paper come from DES and CLASH. The Dark Energy Survey (DES) is an opticalnear-infrared survey that is imaging $5000 \mathrm{deg}^{2}$ of the South Galactic Cap in the griz $Y$ bands over 525 nights spanning 5 years. The survey is being carried out using a new $\sim 3 \mathrm{deg}^{2}$ CCD camera (the DECam; see Flaugher et al. 2015) mounted on the Blanco 4-m telescope at the Cerro Tololo Inter-American Observatory (CTIO) in
Chile. DES started in 2012 with a testing period (2012 November2013 February) called DES Science Verification (SV). ${ }^{1}$ At the time of writing, two observing seasons (Diehl et al. 2014) have been completed, and a third is underway.

The survey strategy is designed to optimize the photometric calibration by tiling each region of the survey with several overlapping pointings in each band. This provides uniformity of coverage and control of systematic photometric errors. This strategy allows DES to determine photometric redshifts of galaxies to an accuracy of $\sigma(z) \simeq 0.07$ out to $z \gtrsim 1$, with some dependence on redshift and galaxy type, and cluster photometric redshifts to $\sigma(z) \sim 0.02$ or better out to $z \simeq 1.3$ (The Dark Energy Survey Collaboration 2005). It will also provide shapes for approximately 200 million galaxies for weak lensing studies. For further information, see The Dark Energy Survey Collaboration (2005) or www.darkenergysurvey.org.

The fact that DECam has an $\sim 3 \mathrm{deg}^{2}$ field of view gives us the opportunity of studying the large-scale structure of galaxy clusters with only one pointing.

The cluster RXJ2248 was observed during the SV season, with typical exposure times of $90 \mathrm{~s}$ for the griz bands and $45 \mathrm{~s}$ for the $Y$ band. It was re-observed later in 2013 to benefit from improvements to telescope performance and general image quality. The data reduction was done using the SVA1 DES Data Management (DESDM) pipeline, described in detail in Sevilla et al. (2011), Desai et al. (2012) and Mohr et al. (2012). The process includes calibration of the single-epoch images, which are then co-added after a background subtraction and cut into tiles. The SVA1 catalogue was created using Source Extractor (SExtractor, Bertin \& Arnouts 1996) to detect objects on the riz co-added images. The median $10 \sigma$ depths of SV data are $g \sim 24.45, r \sim 24.30, i \sim 23.50, z \sim 22.90$, $Y \sim 21.70$, which reach close to the expected DES full depths. Limiting magnitudes were estimated for the $200 \mathrm{deg}^{2}$ SPT-E part of the wide-field SV area using BALROG (Suchyta et al. 2016) and PSF magnitude errors for true point sources.

We use AB magnitudes throughout this paper, and MAG_AUTO measurements given by SEXTRACTOR, as these proved to be robust and were thus used in several DES SV papers (e.g. Bonnett et al. 2015; Crocce et al. 2016). The objects selected for the analysis have a signal-to-noise ratio $(\mathrm{S} / \mathrm{N})>10$ in the $i$ band.

The other survey considered here is CLASH (Postman et al. 2012), a 524-orbit HST multi-cycle treasury programme that has observed 25 massive clusters, having a range of virial masses between $5 \times 10^{14} \mathrm{M}_{\odot}$ to $30 \times 10^{14} \mathrm{M}_{\odot}$ and an average redshift of $\bar{z}=0.4$. The wavelength range covers the UV, the visible and the IR (2000-17 $000 \AA$ ) through 17 bands using the Advanced Camera for Surveys (ACS) and the Wide Field Camera 3 (WFC 3).

The CLASH mosaics were produced using the 'MosaicDrizzle' pipeline (see Koekemoer et al. 2011). The CLASH catalogue creation pipeline makes use of SEXRACTOR: the software is run in dual image mode, where a detection image is created from a weighted sum of the ACS/WFC and WFC3/IR images. The WFC3/UVIS images are not used in the construction of the detection image but the UVIS data are still used to compute source photometry. The photometry given in the public catalogue (http://www.stsci.edu/postman/CLASH), which is also the one used in this work, was measured in isophotal apertures, as they have been shown to produce reliable colours (Benítez et al. 2004). ACS/WFC3 reach a depth of 26.8, 26.4, 26.2, 26.0 and $26.6(10 \sigma$ galaxy AB magnitudes for circular apertures of 0.4 arcsec in diameter; Postman

\footnotetext{
${ }^{1}$ For public data release see: http://des.ncsa.illinois.edu/releases/sva1
} 
Table 1. Main properties of the cluster RXC J2248-4431. The quoted luminosity is in the rest frame $0.1-2.4 \mathrm{keV}$ band.

\begin{tabular}{cccc}
\hline$\alpha_{\mathrm{J} 2000}$ & $\delta_{\mathrm{J} 2000}$ & Redshift & Luminosity $\left(\mathrm{erg} \mathrm{s}^{-1}\right)$ \\
\hline $22: 48: 44.29$ & $-44: 31: 48.4$ & 0.348 & $3.08 \times 10^{45}$ \\
\hline
\end{tabular}

et al. 2012) in the F475W, F625W, F775W, F850LP and F105W filters, respectively.

Below, we compare the information obtained with five DES filters and with 17 HST filters.

\section{THE CLUSTER RXC J2248.7-4431}

In this section we present what is known about this cluster from previous works. The cluster of galaxies RXC J2248.7-4431, where RXC stands for ROSAT X-ray Cluster, is also known as Abell S1063 or MACS 2248-4431. It is a very luminous cluster, having an X-ray bolometric luminosity of $(6.95 \pm 0.1) \times 10^{45} \mathrm{erg} \mathrm{s}^{-1}$ in the energy range $0.1-100 \mathrm{keV}$ (Maughan et al. 2008). Its properties are listed in Table 1, while the experimental specifications of surveys that have observed RXJ2248 are quoted in Table 2. It was first catalogued by Abell, Corwin \& Olowin (1989), who counted 74 galaxies. Thanks to the ROSAT-ESO Flux Limited X-ray (REFLEX) Galaxy Cluster survey, Böhringer et al. (2004) measured a spectroscopic redshift $z=0.3475$, which has been adopted in the recent literature and has also been confirmed in Gómez et al. (2012), who quoted a mean redshift of $z=0.3461_{-0.0011}^{+0.0010}$ for 81 members.

Gómez et al. (2012) were the first to study in detail RXJ2248, even though it is the second most luminous cluster in the REFLEX survey (having a reported luminosity of $\sim 3.08 \times 10^{45} \mathrm{erg} \mathrm{s}^{-1}$ in the rest frame $0.1-2.4 \mathrm{keV}$ band).

In Gómez et al. (2012), the cluster is presented as one of the hottest X-ray clusters known at that time. The high X-ray temperature, together with the high velocity dispersion, suggests a very massive cluster $\left(M_{200 c}>2.5 \times 10^{15} \mathrm{M}_{\odot}\right)$ and/or a merger system. The merger model is supported by a small offset between the galaxy distribution and the peak of X-ray isophotes, and a non-Gaussian galaxy velocity distribution. Gómez et al. (2012) also reported that the velocity distribution is better represented by the velocity dispersion produced during a merger than by the velocity distribution of a relaxed cluster.

Gruen et al. (2013) used Wide-Field Imager (WFI) data to perform a weak lensing analysis of the cluster. They parametrized the cluster density with a NFW profile (Navarro, Frenk \& White 1996) and obtained a mass $M_{200 m}=33.1_{-6.8}^{+9.6} \times 10^{14} \mathrm{M}_{\odot}\left(\right.$ or $M_{200 \mathrm{c}}=$ $\left.22.8_{-4.7}^{+6.6} \times 10^{14} \mathrm{M}_{\odot}\right)$. They also identified a second galaxy cluster in the field of view at redshift $\sim 0.6$, with an estimated mass of $M_{200 m}=4.0_{-2.6}^{+3.7} \times 10^{14} \mathrm{M}_{\odot}$.

Melchior et al. (2015) studied the weak lensing masses and galaxy distributions of four massive clusters observed during the DES $\mathrm{SV}$ period, including RXJ2248. They found $M_{200 \mathrm{c}}=17.5_{-3.7}^{+4.3} \times$ $10^{14} \mathrm{M}_{\odot}$, which is in agreement with previous mass estimates. For RXJ2248, they also identified filamentary structures of the luminous red-sequence galaxies found with the REDMAPPER (Rykoff et al. 2014) algorithm.

Umetsu et al. (2016) combined HST and wide field imaging (from the Subaru telescope or the ESO/WFI) observations to reconstruct the surface mass density profiles of 20 CLASH clusters. Their analysis jointly uses strong lensing as well as weak lensing with shear and magnification, and for RXJ2248 they found $M_{200 \mathrm{c}}=18.78 \pm 6.72 \times 10^{14} \mathrm{M}_{\odot}$.

\section{COMPARISON OF DES AND CLASH}

In this section, we assess detectability, photometry, and stellar masses of DES galaxies, treating matched CLASH galaxies as truth table. ${ }^{2}$ In order to make the comparison, we seek to identify similar filters in both data sets. Fig. 1 shows that the closest HST analogues to DES griz are F475W, F625W, F775W and F8501p. We will refer to the corresponding $H S T$ and DES bands as $g, r, i$ and $z$ for simplicity of notation. In the following, we will also use the DES $Y$ band, which does not have a similar HST filter. When we refer to five CLASH filters, it means we are including the F105W filter, which is broader than the DES $Y$.

In the DES catalogue of the RXJ2248 area, there are 374294 sources in a roughly circular area of approximately $3 \mathrm{deg}^{2}$. The deeper, higher resolution CLASH catalogue includes 3471 sources in a much smaller area $\left(\sim 5 \times 4 \operatorname{arcmin}^{2}\right)$.

We perform a spatial matching (using a matching radius of 1.5 arcsec) between the DES and CLASH catalogues and we find 609 matched sources. Thus, the DES recovered only 18 percent of the sources in the CLASH catalogue. The high percentage of sources missed in DES is due to various problems, one of them being that the griz $10 \sigma$ depths differ by $\gtrsim 2$ mag between the two data sets. This accounts for most of the undetected sources in DES: when we simulate fake faint galaxies with $\mathrm{BALROG}^{3}$ (Suchyta et al. 2016) on the DES image of RXJ2248, we find that the completeness in riz bands (which are those used to run the detection) drops below 20 per cent between magnitude 24 and 25, justifying the incompleteness found when comparing to the even deeper CLASH survey. We also expected one of the problems to be blending, especially close to the bright cluster core. We run some completeness tests using a DES enhanced deblending catalogue (Zhang et al. 2015) that would increase the percentage of recovered sources to 20 per cent, but found that blending is not a major reason of incompleteness. Also, CLASH object detection is run on ACS+IR images, while DES detection only involves optical bands and it may miss redder sources. A visual comparison of DES and CLASH images is shown in Fig. 2.

A comparison of measured isophotal magnitudes at the catalogue level between the matched galaxies in the two data sets here considered shows a mean shift $|\Delta m| \leq 0.13$ in all bands, where the offsets due to the different filters compared have been taken into account. This is true when a signal-to-noise ratio cut $\mathrm{S} / \mathrm{N}>10$ is performed on the matched galaxies, and objects with saturated pixels and corrupted DES data are removed. A magnitude analysis is presented in Appendix A.

\subsection{Star/galaxy separation}

For the purpose of studying the star/galaxy separation, we adopt the same notation used in Soumagnac et al. (2015). We study the galaxy completeness $c_{\mathrm{g}}$, defined as the ratio of the number of true

\footnotetext{
${ }^{2}$ A comparison of weak lensing measurements between DES and CLASH was not performed because they predominantly reveal differences in the shear calibration. The majority of galaxies with shape measurement in both catalogues are very faint for DES, resulting in large and noisy calibration factors (see section 4.2.1 in Melchior et al. 2015). In addition, the high density of galaxies in the central region of this cluster creates many more close galaxy pairs or even blends in ground-based DES images than when viewed with $H S T$, rendering shape measurement even more challenging. A detailed analysis of those relevant effects is beyond the scope of this paper. ${ }^{3}$ A software pipeline for embedding simulations into astronomical images. See: https://github.com/emhuff/Balrog.
} 
Table 2. Some experimental specifications of the surveys that have observed RXJ2248, with the corresponding paper in which those data have been used. The work presented in those papers is briefly summarized in Section 3. The magnitude limits reported for DES are the mean $10 \sigma$ galaxy magnitudes.

\begin{tabular}{|c|c|c|c|c|c|c|}
\hline Survey/Instrument & Authors & FoV & Filters & Mag limits & Spectra & Objects \\
\hline CLASH & $\begin{array}{l}\text { Postman et al. (2012), } \\
\text { Monna et al. (2014) }\end{array}$ & $\begin{array}{c}3.4 \times 3.4 \operatorname{arcmin}^{2}(\mathrm{ACS}) \\
2 \times 2 \operatorname{arcmin}^{2}(\mathrm{WFC} 3)\end{array}$ & 16 in $2000-17000 \AA$ & $\sim 25-27(10 \sigma)$ & - & 3471 \\
\hline WFI & Gruen et al. (2013) & $33 \times 33 \operatorname{arcmin}^{2}$ & UBVRIZ & $\begin{array}{l}26.4,26.7,24.4 \\
\quad(\text { VRI } 5 \sigma)\end{array}$ & - & - \\
\hline DES SV & Melchior et al. (2015) & $2.2 \mathrm{deg}^{2}$ & $\operatorname{griz} Y$ & $\begin{array}{c}24.45,24.30,23.50, \\
22.90,21.70(10 \sigma)\end{array}$ & - & 374294 \\
\hline
\end{tabular}

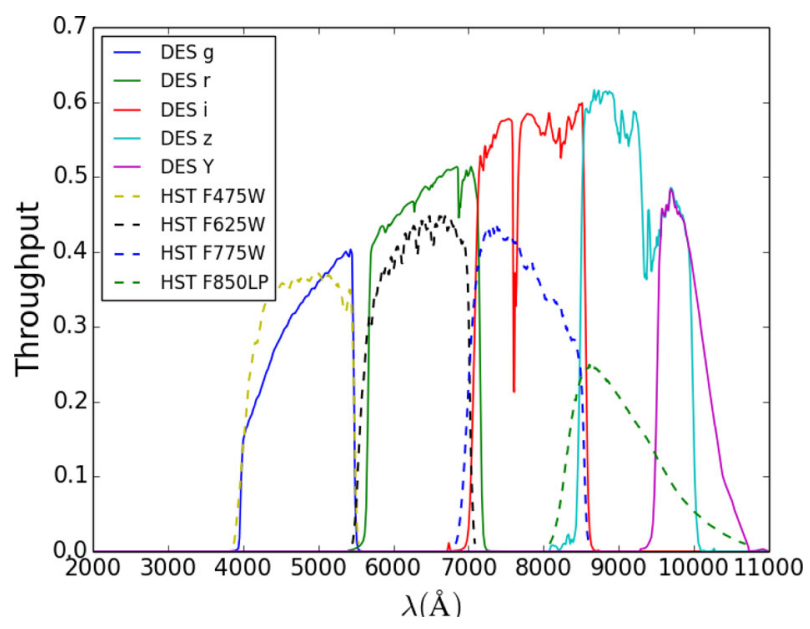

Figure 1. Throughput of the DES filters (solid lines) and HST similar filters (dashed lines).

galaxies classified as galaxies to the total number of true galaxies (including then also the number of true galaxies classified as stars $\left.M_{\mathrm{G}}\right)$ :

$c_{\mathrm{g}}=\frac{N_{\mathrm{G}}}{N_{\mathrm{G}}+M_{\mathrm{G}}}$,

where here $N_{\mathrm{G}}$ is given by the galaxies in the DES catalogue, and the number of true galaxies is given by the object classified as such in CLASH.

Moreover, the galaxy purity $p_{\mathrm{g}}$ is defined as

$p_{\mathrm{g}}=\frac{N_{\mathrm{G}}}{N_{\mathrm{G}}+M_{\mathrm{S}}}$,

where $M_{\mathrm{S}}$ is the number of stars classified as galaxies.

We consider as true galaxies the sources that have a SEXTRACTOR stellarity index CLASS_STAR $<0.08$ in the CLASH catalogue, otherwise they are stars. This cut has been proven to perform well in other CLASH works (e.g. Jouvel et al. 2014). We try to understand if the star/galaxy performance is compatible between the two data sets.

We first consider the CLASS_STAR parameter given in the DES catalogue. We find that a cut between 0.7 and 0.9 for the CLASS_STAR_I gives purity and completeness above 90 per cent. The number of galaxies and stars in the two catalogues can be found in Table 3.

We also test the performance of star/galaxy separation with the SPREAD_MODEL parameter (defined in Desai et al. 2012 and tested in Bouy et al. 2013). SPREAD_MODEL is a morphological
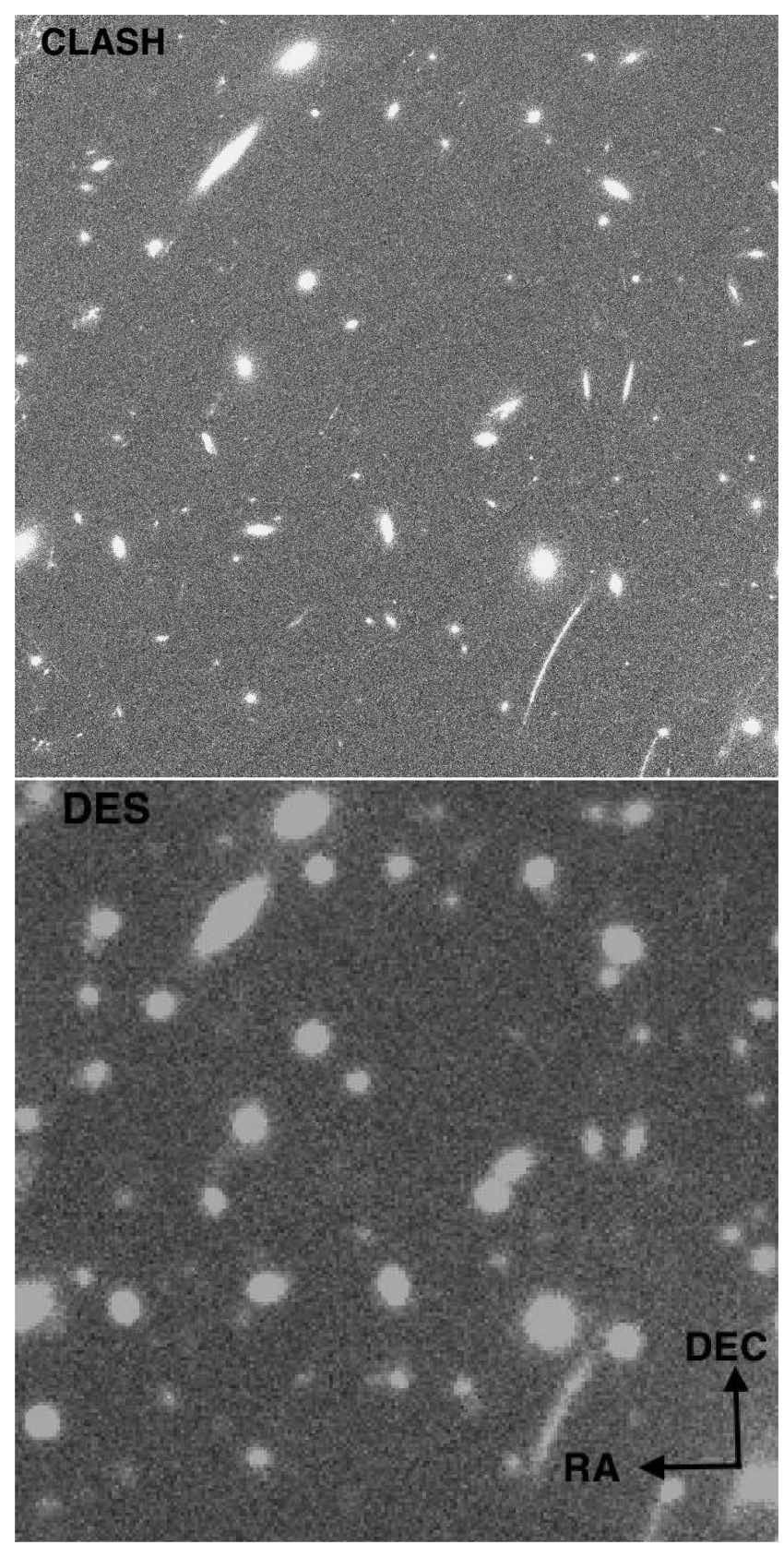

Figure 2. A portion of $1 \times 1 \operatorname{arcmin}^{2}$ image centred in RA 22:48:48.003 and DEC -44: 31 : 38.52 in the CLASH F625W band (top) and the DES $r$ band (bottom). 
Table 3. Number of galaxies $G$ and stars $S$ found in DES and CLASH, when considering CLASS_STAR $<0.8$ (top table) and SPREAD_MODEL $>0.003$ (bottom table) for galaxies in DES.

\begin{tabular}{lcccc}
\hline CLASS_STAR & $g$ & $r$ & $i$ & $z$ \\
\hline$G($ DES $)$ & 551 & 538 & 535 & 522 \\
$G($ CLASH) & 553 & 553 & 553 & 553 \\
$S($ DES $)$ & 58 & 71 & 74 & 87 \\
$S($ CLASH) & 56 & 56 & 56 & 56 \\
SPREAD_MODEL & $g$ & $r$ & $i$ & $z$ \\
$G($ DES $)$ & 388 & 463 & 428 & 405 \\
$G($ CLASH) & 553 & 553 & 553 & 553 \\
$S($ DES $)$ & 221 & 146 & 181 & 204 \\
$S($ CLASH $)$ & 56 & 56 & 56 & 56 \\
\hline
\end{tabular}

star/galaxy separation parameter given by SEXTRACTOR which acts as a linear discriminant between the best-fitting local PSF model and a slightly 'fuzzier' version made from the same PSF model, convolved with a circular exponential model. A threshold is set to 0.003 by the DESDM pipeline to separate stars (PSF like, having absolute values below 0.003) from galaxies (non-PSF like, with values higher than 0.003). As a result, 77.4 per cent of the galaxies are catalogued in DES as such, and the purity is 97.3 per cent. A plot for the purity and the completeness for varying SPREAD_MODEL_I cuts is shown in Fig. 3. We list the number of galaxies and stars in the two catalogues in Table 3. It can be seen from Fig. 3 that cut at lower values $(\sim 0.001-0.002)$ would give a higher completeness without affecting the purity significantly. Moreover, this case may be better using the SPREAD_MODEL in the $r$ band, which is deeper than the $i$ one, and this can also be seen in Fig. 3, where it is clear that, for the same cut, the completeness is higher. We also find that using the CLASS_STAR parameters with the mentioned cut is more efficient than adopting the SPREAD_MODEL_I with the cut at 0.003 .

\subsection{Photo- $z$}

Considering only those matched sources with a $\mathrm{S} / \mathrm{N}>10$ in the DES $i$-band, excluding stars (in this case we exclude all objects with CLASS_STAR_I $>0.8$ ) and objects with FLAGS $\neq 0$ (in order to exclude objects with saturated pixels or corrupted data, and originally blended sources) we are left with 155 sources. This is the subset of galaxies that we will use for the photo- $z$ and stellar mass comparison

In order to estimate the photo- $z \mathrm{~s}$, we used the publicly available software LePhare (Arnouts et al. 1999; Ilbert et al. 2006), ${ }^{4}$ as it also produces the stellar masses that we want to study in this paper. Previous works on DES photo- $z$ s have tested the performance of this code in comparison with other software and spectroscopic redshifts. In particular, Sánchez et al. (2014) found that LePhARE fulfils the DES requirements on scatter and $2 \sigma$ outlier fraction when it is run on SV data, and the metrics obtained are compatible with those from other template-based methods within 10 percent. Training-based methods showed a lower bias compared to template-fitting codes, and this can be improved in the latter using adaptive recalibration methods, which are available in LePhARE. However, stellar mass tests have not been performed with DES data so far.

\footnotetext{
${ }^{4}$ http://www.cfht.hawaii.edu/arnouts/LEPHARE/lephare.html
}
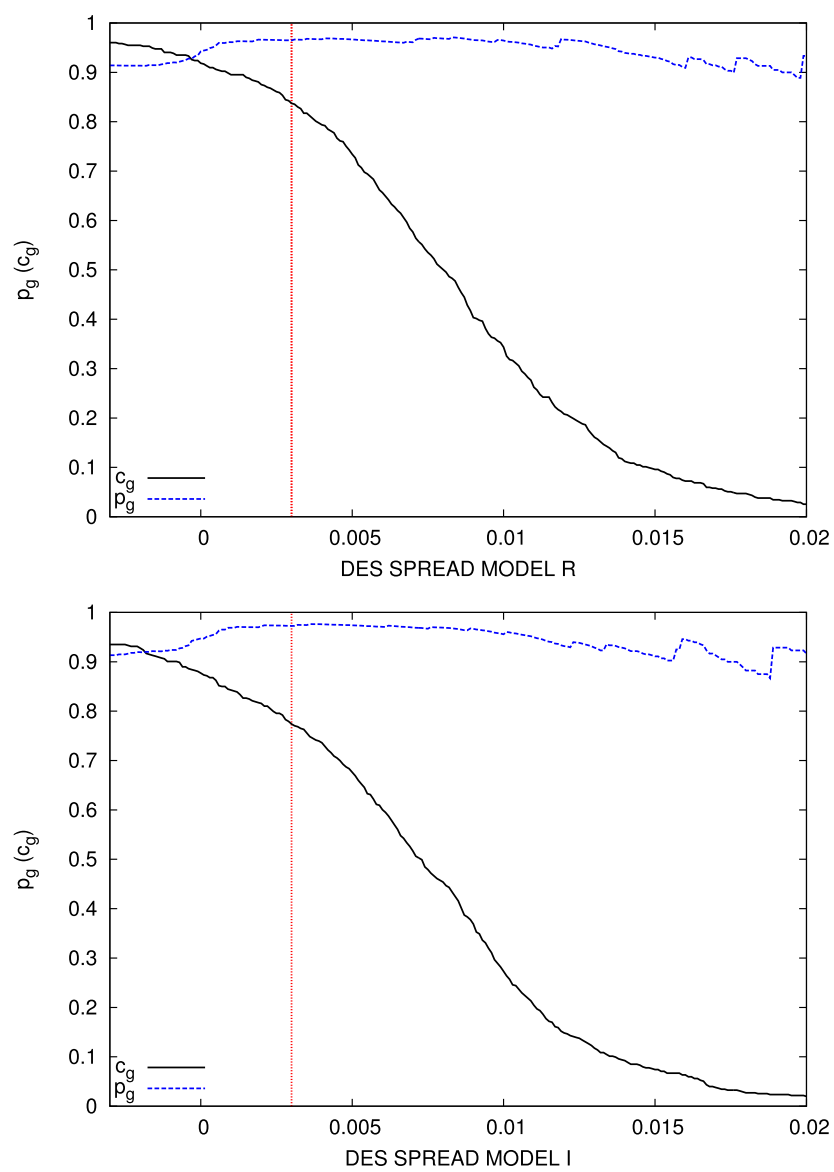

Figure 3. Galaxy purity (blue dashed line) and completeness (black solid line) for the star/galaxy separation problem using the SPREAD_MODEL parameter in the DES catalogue for the $r$ (top) and $i$ (bottom) bands. The red vertical line represents a typical cut used for SPREAD_MODEL, which is 0.003 .

We therefore need to further check the DES photo- $z$ and stellar mass estimation with LEPHARE first. This is where the HST data are particularly useful in this work, as we need to check DES against a more precise photometric survey covering the wavelengths from optical to IR.

Furthermore, LePhare is a reliable code, as seen in e.g. Ilbert et al. (2009).

\subsubsection{LePHARE photo-z technique}

The main purpose of LEPHARE is to compute photometric redshifts by comparing template Spectral Energy Distributions (SEDs) to the observed broad-band photometry, but it can also be used to calculate physical parameters such as stellar masses and rest-frame luminosities. Several SED sets are available within the code, and these are redshifted and integrated through the instrumental transmission curves. Additional contribution of emission lines in the different filters can be included and extinction by dust can be taken into account. The synthetic colours obtained from the SEDs for each redshift are then compared to the data. The best-fitting template and redshift for each object is then found by $\chi^{2}$ minimization. In addition, prior information can be supplied, including a photo- $z$ distribution prior by galaxy type computed from the VVDS survey in the $i$ band (see Ilbert et al. 2006). 


\subsubsection{Results}

We run LePhARE on both CLASH (with five, eight and all 17 filters) and DES (five filters) catalogues, fitting the 31 synthetic SEDs templates given by the COSMOS (see Ilbert et al. 2009) libraries. We use four galaxy extinction values ranging from 0.05 to 0.3 using a Calzetti et al. (2000) extinction law for DES. For CLASH, two more extinction values are added $(0.4$ and 0.5$)$ in order to take into account the wider wavelength range covered when we use all its filters.

The results are plotted in the upper panel of Fig. 4 for the 155 matched sources when five filters are considered for CLASH. Of all the sources considered, 85 per cent have a photo- $z$ which is compatible with the CLASH photo- $z$ within the DES requirement ${ }^{5}$ $\left|z_{\mathrm{p}}-z_{\mathrm{s}}\right|<\sigma\left(1+z_{\mathrm{s}}\right)$, where $\sigma=0.12$. We notice an offset in the CLASH redshift when 17 filters are used (see lower panel of Fig. 4), while 77 per cent of the sources still satisfy the DES requirement. This most likely stems from the inclusion of near-UV filters to get an accurate redshift from the Balmer break for galaxies below a redshift of 0.4 (see e.g. Eisenstein et al. 2001). In fact, this offset starts to be seen also when adding only three UV bands (namely F336W, F390W and F435W) to the grizY filters (see middle panel in Fig. 4). A problem around redshift 0.4 for DES galaxies had already been seen in Sánchez et al. (2014) (see their fig. 5) and Bonnett et al. (2015). In particular, Bonnett et al. (2015) also pointed out a lack of matching SEDs for galaxies around redshift 0.4 with template fitting methods (see their fig. 8).

Zero-points ${ }^{6}$ have not been adopted in the DES photo- $z$ estimation, as we saw that their introduction causes systematic effects. Zero-points are calculated using field galaxies, so we believe we would need spectroscopy in the cluster field to be helpful at photo- $z$ calibrations for this study.

\subsection{Stellar masses}

Stellar masses are key observables in the study of galaxy evolutionary models. Unfortunately, they cannot be directly measured, but require multicolour photometry to be fitted with stellar population models, therefore making a series of assumptions. One of these is the galaxy redshift if spectroscopy is not available: in the view of our goal of computing the stellar mass profile of the RXJ2248 cluster, we have to bear in mind that galaxy redshift accuracy is essential not only to ensure the correct template match in the template fitting method here used and the distance to the galaxy, but also to determine the cluster membership. We will therefore see how the redshift assumptions affect the stellar mass estimation and elaborate a reasonable technique to correctly estimate the stellar mass profile.

\subsubsection{Method}

We use the same sample of matched galaxies with $\mathrm{S} / \mathrm{N}>10$ used in Section 4.2 and their redshift estimations in order to compute the stellar masses for both DES and CLASH using LEPHARE. In the first place, the redshifts of the galaxies are fixed to those photo- $z \mathrm{~S}$ previously computed (i.e. to DES photo- $z$ s for DES stellar masses, and to CLASH photo- $z$ s for CLASH stellar masses). In the second

\footnotetext{
${ }^{5}$ Where $z_{\mathrm{p}}$ and $z_{\mathrm{s}}$ are the photometric and spectroscopic redshifts, so here we consider the CLASH photo- $z$ as the "spectroscopic" one.

${ }^{6}$ Zero-points define the shift in the observed magnitudes due to various systematics.
}
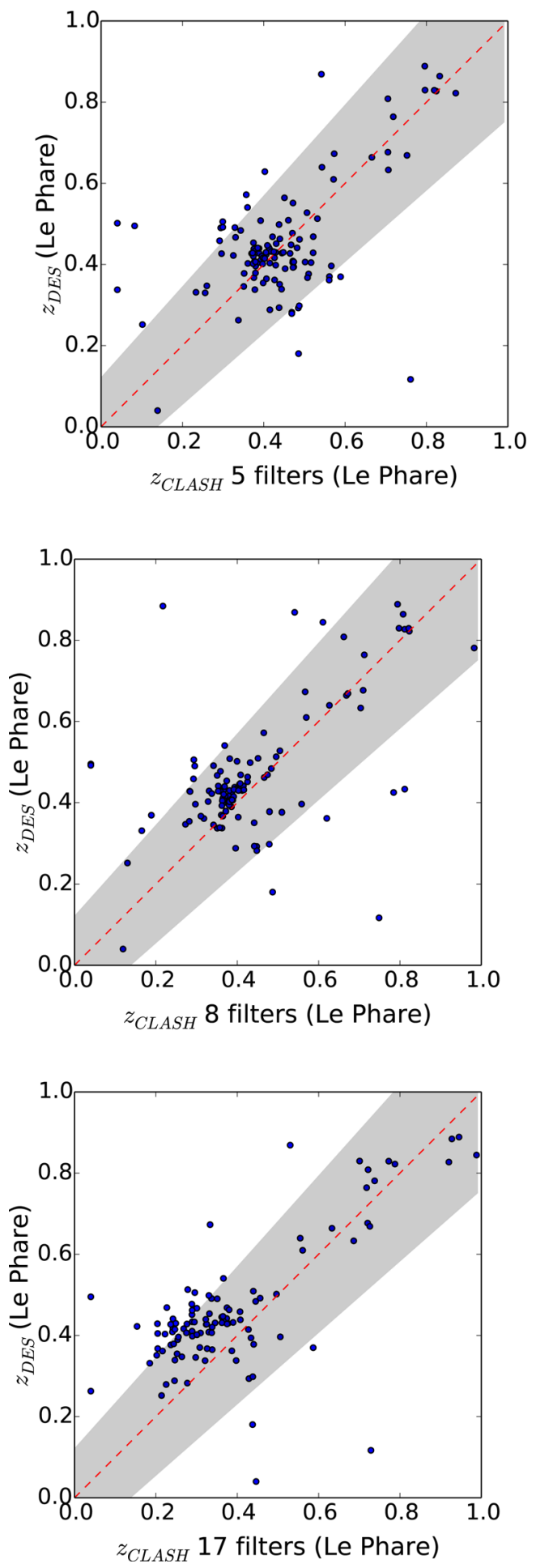

Figure 4. Comparison of the DES versus CLASH photo- $z$ s for the sources matched between the two catalogues. photo- $z$ s were obtained using LePhare. Top: only the 5 HST filters similar to the grizY filters in DES have been used to compute CLASH photo-zs. Middle: three of the HST UV filters have been added to the five HST optical filters for the CLASH photo- $z$ estimation. Bottom: all the 17 available CLASH filters have been used to estimate the photo- $z$ s. The red dashed line represents $z_{\mathrm{DES}}=z_{\mathrm{CLASH}}$, and the grey area the expected DES accuracy of $\left|z_{\mathrm{DES}}-z_{\mathrm{CLASH}}\right|<\sigma\left(1+z_{\mathrm{CLASH}}\right)$, where $\sigma=0.12$. 

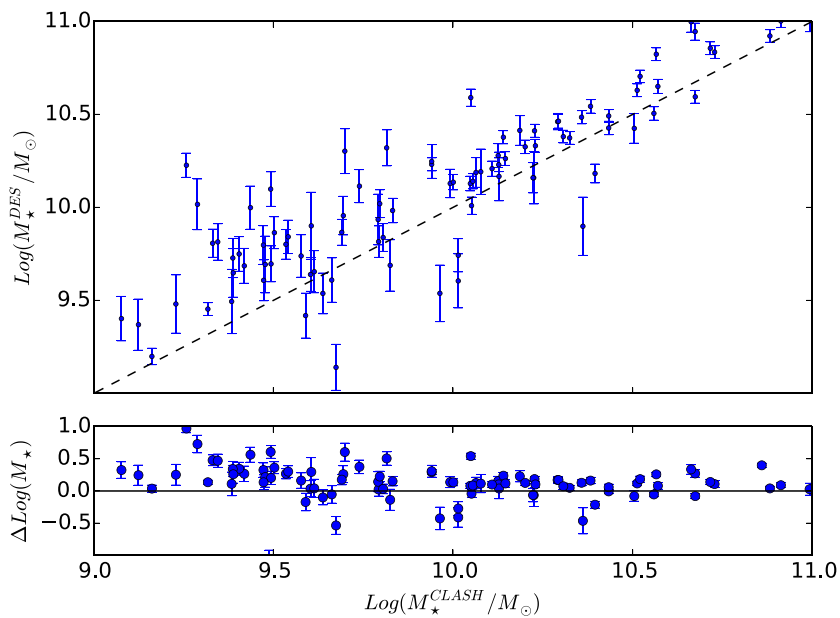

Figure 5. DES stellar masses versus CLASH stellar masses computed using LePhARE. In the stellar mass estimation, each source is assumed to be at the redshift given as output by LePhare, as described in Section 4.2. The dashed line represents $M_{\star}^{\mathrm{DES}}=M_{\star}^{\mathrm{CLASH}}$. In the bottom panel $\Delta \log \left(M_{\star}\right)=\log \left(M_{\star}^{\text {DES }}\right)-\log \left(M_{\star}^{\text {CLASH }}\right)$ is presented. All available filters (i.e. five for DES and 17 for CLASH) have been used in the estimation process. Uncertainties represent the 68 per cent Confidence Level.

case, we fix the galaxy redshifts at the cluster redshift for both DES and CLASH, and the LEPHARE DES photo-zs are only used to select a subsample of cluster members satisfying $\left|z_{\text {phot }}-z_{\mathrm{cl}}\right| \leq 0.12$. For this subsample both DES and CLASH stellar masses are estimated.

We chose to use LePhare, together with the Bruzual \& Charlot (2003) templates, as this combination has been shown to be robust in the estimation of physical parameters of galaxies (Ilbert et al. 2010).

We derive our stellar mass estimates by fitting synthetic SEDs templates while keeping the redshift fixed as described previously in the two cases. The SED templates are based on the stellar population synthesis (SPS) package developed by Bruzual \& Charlot (2003) (BC03) assuming a Chabrier (2003) initial mass function (IMF). Our initial set of templates includes nine models using one metallicity $\left(Z=1 Z_{\odot}\right)$ and nine exponentially decreasing star formation rates $\propto \exp (-t / \tau)$ where $t$ is the time and $\tau$ takes the values $\tau=0.1,0.3$, $1,2,3,5,10,15,30$ Gyr. The final template set is then generated over 57 starburst ages ranging from 0.01 to $13.5 \mathrm{Gyr}$, and four extinction values ranging from 0.05 to 0.3 using a Calzetti et al. (2000) extinction law. For CLASH, two more extinction values are added $(0.4$ and 0.5$)$.

The uncertainties on our stellar masses estimates (MASS_BEST from LEPhARE) are given by the 68 per cent confidence limits on the SED fit.

\subsubsection{Results}

In Fig. 5, we show the comparison between DES and CLASH stellar mass estimates for the first case, where the redshifts are fixed to the LEPhARE estimates. The linear correlation between the two estimates is clear, but there is an offset of mean value $\sim 0.16 \mathrm{dex}$. This should be considered in light of two aspects:

(i) the offset in the photo- $z$ s that we addressed in Section 4.2;

(ii) the uncertainties in the DES stellar masses may be underestimated as those are the 68 per cent confidence limits on the SED fit and do not take into account systematic error contribution.
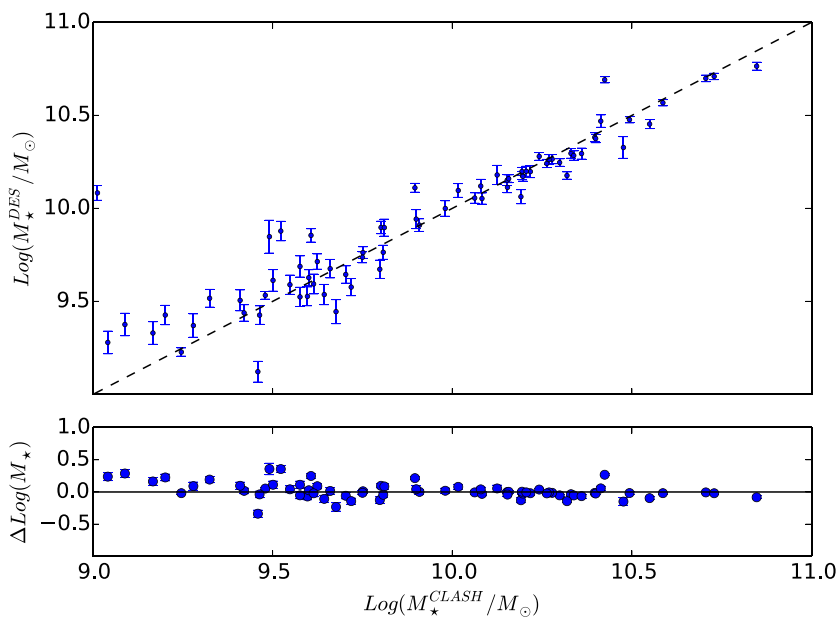

Figure 6. DES stellar masses versus CLASH stellar masses computed using LEPHARE. Here only sources around the cluster redshift are considered (i.e. sources with a DES photo- $z$ that satisfies $\left|z-z_{\mathrm{cl}}\right| \leq 0.12$, where $z_{\mathrm{cl}}=0.3475$ is the cluster redshift). In the DES and CLASH stellar mass estimation, these galaxies are all assumed to be at $z_{\mathrm{cl}}$. The dashed line represents $M_{\star}^{\mathrm{DES}}=$ $M_{\star}^{\mathrm{CLASH}}$. In the bottom panel $\Delta \log \left(M_{\star}\right)=\log \left(M_{\star}^{\mathrm{DES}}\right)-\log \left(M_{\star}^{\mathrm{CLASH}}\right)$ is presented. The offset seen in Fig. 5 seems to disappear in this plot, showing that this effect was due to the photo- $z$ offset. All available filters (i.e. five for DES and 17 for CLASH) have been used in the estimation process. Uncertainties represent the 68 per cent Confidence Level.

In Fig. 6 we show the results for the second case, where we select the galaxies with a DES photo- $z$ close to the spectroscopic cluster redshift $z_{\mathrm{cl}}$, satisfying $z_{\mathrm{cl}}-0.12<z_{\text {phot }}<z_{\mathrm{cl}}+0.12$. The redshift of these sources is fixed at $z_{\mathrm{cl}}$ in the stellar mass estimation, and the reason for this choice is twofold:

(i) to minimize circularity associated with using LEPHARE to both measure redshifts and stellar masses;

(ii) to take into account the shift in the redshift estimates pointed out in Section 4.2 (and therefore put at the correct cluster redshift the cluster members which photo- $z$ appeared to be at $z_{\text {phot }} \sim 0.4$ ).

Of course, this choice results in considering some sources as being at $z_{\mathrm{cl}}$ even though they are not, and we shall take this into account in the following. A consistency test for the choice of redshifts done is presented in Appendix B.

The correlation between the estimated stellar masses significantly increases if also the CLASH sources are set to be at the cluster redshift, as seen in a comparison of Fig. 5 with Fig. 6, where we find that stellar masses from DES can be estimated within 25 percent of CLASH values. This shows that the offset seen in the former is due to the offset in the redshifts given in input, rather than other systematics. Therefore, the wavelengths covered by the DES broadband filters are capable of providing a good estimation of stellar mass if the photometric redshift is sufficiently precise.

In Fig. 7, we show the spatial distribution of the total stellar mass spatial distributions for both DES and CLASH in the CLASH field $\left(\sim 4.8 \times 4.2 \operatorname{arcmin}^{2}\right)$, represented with a resolution of 0.12 arcmin/pixel and smoothed with a Gaussian of $\sigma=0.144$ arcmin. The stellar masses of galaxies are summed over in each pixel. Obviously, the two samples show very good agreement in terms of the spatial distribution of stellar mass. The Pearson coefficient for the pixel-by-pixel stellar mass values of the two non-smoothed maps is 0.93 . The difference map without any smoothing has a mean of 0.02 and $\sigma=0.12$ 


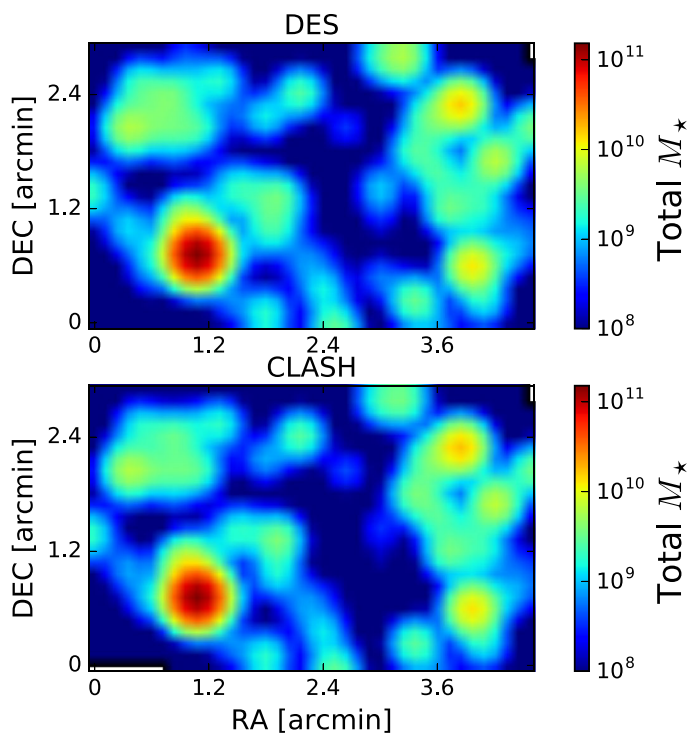

Figure 7. DES and CLASH total stellar mass maps computed using LePhare for the galaxies matched between the two catalogues. The stellar masses plotted are the same as those shown in the bottom panel of Fig. 6 (i.e. all the galaxies with a DES photo- $z$ that satisfies $\left|z-z_{\mathrm{cl}}\right| \leq 0.12$, where $z_{\mathrm{cl}}$ is the cluster redshift, have a redshift fixed to $z_{\mathrm{cl}}$ in the SED fitting). The map is centred on the BCG, but its stellar mass is not visible as it was originally blended and therefore did not pass the quality flag cut applied in Section 4.3. The resolution is $0.12 \mathrm{arcmin} / \mathrm{pixel}$ and the map is smoothed with a Gaussian of $\sigma=0.144 \mathrm{arcmin}$. At the cluster redshift, $1 \mathrm{arcmin}$ corresponds to $294 \mathrm{kpc}$ in the assumed cosmology.

\section{DARK MATTER AND STELLAR MASSES}

In this section we study the stellar mass radial profile of the cluster and relate it to that of dark matter that has been obtained though DES weak lensing studies in Melchior et al. (2015). As shown in Section 4.3, stellar mass estimation can be biased if the redshift assumed is biased too: this is a problem if one wants to compute stellar masses with photometric surveys data. In the lack of spectroscopy, it is a common method to perform estimation of the photometric redshift and SED fitting in two steps, which involve fixing the redshift of a galaxy at the best-fitting value obtained in the first step. Although this may not be the most elegant way of solving the problem, it has been proven to lead to only a small bias in the SED fitting parameters, when compared to results given by the simultaneous estimation of redshift and stellar mass (see Acquaviva, Raichoor \& Gawiser 2015). Nevertheless, here we want to adopt a more consistent methodology for the stellar mass estimation, taking advantage of the fact that we are looking at a cluster with a known redshift. This technique is outlined in the first part of this section, followed by a study of the different mass radial profiles obtained. To allow a straightforward comparison with the weak lensing reconstructed mass, we compute total stellar mass and surface density on a projected two-dimensional plane, i.e.:

$M_{\star}(R)=\sum_{i} m_{\star}^{i} \quad \Sigma_{\star}(R)=\frac{\sum_{i} m_{\star}^{i}}{A_{\text {annulus }}}$,

where the sums are intended over the galaxies within annuli of projected radius $R$. Similar definitions apply for the cumulative distributions $M_{\star}(<R)$ and $\Sigma_{\star}(<R)$, computed within circles of radius $R$. The centre of the image is taken to be that of the BCG. At last, we present a comparison between the stellar and total DES mass maps.

\subsection{Galaxy samples and stellar mass estimates}

Our goal is to compare the reconstructed mass from weak lensing to the total stellar mass of the cluster members. In order to do so, we split the galaxies into two populations. The following steps are performed.

(i) We select the red members using the REDMAPPER SV catalogue (Rykoff et al. 2016), which identifies cluster members with high precision (Rozo et al. 2015). Their stellar masses are computed using the same parameters presented in Section 4.3 (but fixing the redshift at $z_{\mathrm{cl}}$ ).

(ii) The RedMAPPER galaxies profile has been corrected by a factor representing the contribution coming from faint sources at luminosities smaller than the limit of the sample $\left(0.2 L^{*}\right)$. This is done by integrating the luminosity with a Schechter function, i.e. we computed the fraction:

$F_{L}=\frac{\int_{0}^{0.2 L^{*}} L \phi(L) d L}{\int_{0}^{\infty} L \phi(L) d L}$,

where $\phi(L)=\phi_{*}\left(\frac{L}{L^{*}}\right)^{\alpha} e^{-L / L^{*}}$ with $\alpha=-1$ [as done in Rykoff et al. (2014) for the SDSS sample that has properties similar to DES]. We find that the galaxies below the luminosity limit contribute to a fraction $F_{L}=0.18$ of the total luminosity, and therefore, assuming a constant $M_{\star} / L$ ratio for the red galaxy population, they contribute to the same percentage of stellar mass.

(iii) The contribution to the total stellar mass of each red member is weighted by its membership probability (reported in the RedMaPPer catalogue).

(iv) In order to study the mass profile at radii higher than $r_{200 \mathrm{c}}$, we decide not to neglect the contribution coming from the bluer population. First, we exclude all objects with saturated pixels or corrupted data, but include galaxies that were initially blended (such as the BCG). Then we select the rest of the galaxies in the field of view that have magnitudes $m_{i}$ in the $i$ band satisfying $m_{i}^{\mathrm{BCG}}<$ $m_{i}<m_{i}^{\text {lim }}$. In this way we exclude any source which is brighter than the BCG and cut at $m_{i}^{\lim }=21 \mathrm{mag}$ in order to ensure the completeness of the sample. After having performed a SED fitting as in the previous step, we filter out all galaxies that do not give a good fit (cutting on reduced $\chi^{2}<2$ ) when the redshift is fixed at the cluster value.

\subsection{Masking and background correction}

We estimate the survey area lost due to masked regions and blending of faint galaxies with large cluster members near the core. We calculate corrections for both effects as follows.

(i) Healpix (Górski et al. 2005) maps of depth and masking fractions are produced for DES with MANGLE (Swanson et al. 2008). From these, we calculate mean depth and fractions of the masked area in our set of annuli. The depth is approximately constant out to $\approx 50$ arcmin from the BCG, which defines the outer limit of the area used for our background estimation scheme. Masking fractions are below 5 per cent for all annuli and applied to the binned stellar mass estimates from both galaxy samples.

(ii) For the blue galaxy sample, some objects are lost due to blending with cluster member galaxies. Without correction, this would bias our stellar mass estimates of blue galaxies near the cluster centre low. We estimate the area lost in each annulus as the isophotal area above the SEXTRACTOR detection threshold, ISOAREA_I. This yields $\mathrm{a} \approx 7$ per cent correction in the innermost arcminute, which drops quickly towards larger radii. For the blue 


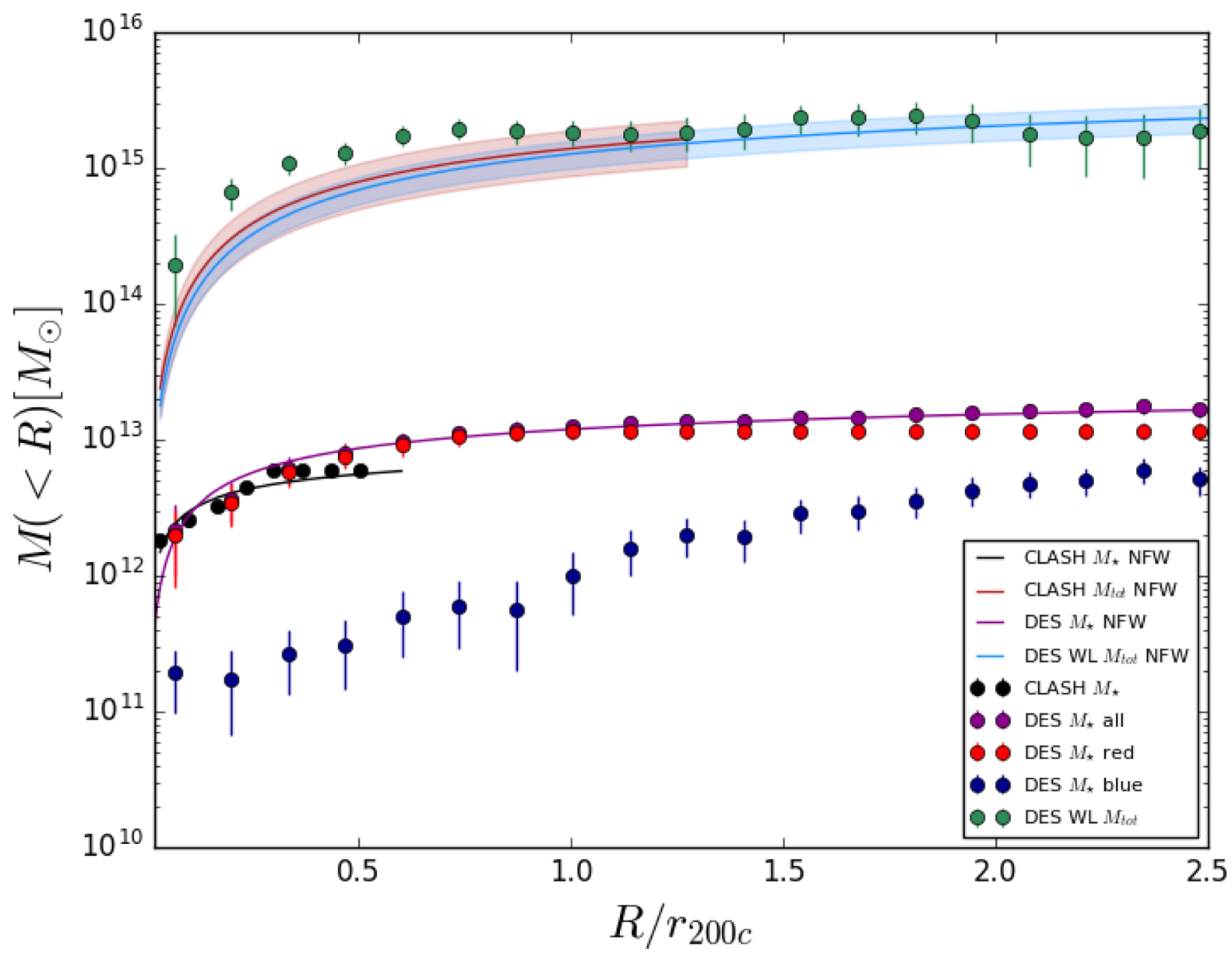

Figure 8. Cumulative radial distributions of total stellar mass derived in this paper for the DES red (red points), blue (blue points) and all galaxies (purple points) in the cluster, together with the total, non-parametric mass profile reconstructed from DES weak lensing (Melchior et al. 2015, green points). The purple solid line is our NFW fit to the DES stellar mass profile, while the blue solid line is the NFW best fit from DES weak lensing. The black points represent the CLASH total stellar mass profile computed in this paper, with our NFW fit (black solid line). The red solid line is the Umetsu et al. (2016) NFW best fit for CLASH from a strong lensing, weak lensing and magnification joint analysis. This profile is restricted to the NFW fitting range $R<2 \mathrm{Mpc} h^{-1}$ chosen in Umetsu et al. (2016), which is larger than the HST field of view as other data sets were used in a joint analysis. The radius $R$ is projected, and $r_{200 c}=2.2 \mathrm{Mpc}$. Error bars show the 68 per cent confidence level.

galaxy sample, this correction and the masking fraction are applied in an additive fashion.

The contribution coming from galaxies that do not actually sit at the cluster redshift is removed from the blue galaxies sample by performing a background subtraction: we estimate the projected surface density of the stellar mass $\Sigma_{\star}(R)$ at large radii (30-50 arcmin, which means outside $\approx 4 r_{200 \mathrm{c}}{ }^{7}$ ), where the stellar mass profile tends to become flat. The value found is $\Sigma_{\star}=1.36 \times 10^{10} \mathrm{M}_{\odot} \operatorname{arcmin}^{-2}$ and this is subtracted on the smaller scales, with an uncertainty given by a Poissonian error. The remaining stellar mass contribution is then added to that of the red galaxies.

\subsection{Stellar mass profile}

We look at the radial distribution of stellar mass, taking into account both the red cluster members present in the REDMAPPER catalogue, and the blue members, as explained in the previous section. The

\footnotetext{
${ }^{7} r_{200 \mathrm{c}}=2200 \mathrm{kpc}$ from the NFW fit of Melchior et al. (2015), which means $r_{200 \mathrm{c}} \simeq 7.46 \mathrm{arcmin}$ at the cluster redshift
}

splitting into red and blue galaxies is justified by the possibility of improving the SED fitting by using different priors for the two populations, and considering the systematics differently. In fact, it is well known that stellar masses estimated for quiescent galaxies are more reliable than for star-forming ones, partially because the colour $-M_{\star} / L$ (from which $M_{\star}$ is derived) relation is more uncertain for very blue colours (see e.g. Conroy 2013; Banerji et al. 2013).

The total stellar mass cumulative profiles $M_{\star}(<R)$ for the red and blue galaxies are shown in Fig. 8. Within the innermost 5 arcmin, the contribution of the red cluster members to the total stellar mass is dominant ( $\gtrsim 80$ per cent) with respect to the bluer galaxies, while at larger radii, namely outside $r_{200 c}$, the second population considered gives a $20 \ldots 50$ per cent contribution to the total stellar mass. In Fig. 8 we also plot the stellar mass profile from CLASH, where the galaxy cluster members were selected cutting on the CLASH photometric redshift with $\left|z_{\text {phot }}-z_{\mathrm{cl}}\right|<0.12$.

\subsection{Comparison to total mass from weak lensing}

The weak lensing mass profile is computed through the aperture mass densitometry (see Clowe et al. 1998) using $M_{\mathrm{tot}}(<R)=$ $\pi R^{2} \zeta(<R) \Sigma_{\mathrm{cr}}\left(z_{1}, z_{\mathrm{s}}\right)$, where $\zeta(<R)=\bar{\kappa}(<R)-\bar{\kappa}\left(r_{1}<r<r_{2}\right)$ is 
the difference between the mean convergence within a circular aperture of radius $R$ and the mean convergence between $r_{1}$ and $r_{2}$ (annulus radii that are fixed for all the apertures in the measurement), $z_{1}$ and $z_{\mathrm{s}}$ are the redshift of lens and sources. The convergence $\kappa$ is defined as the projected surface mass density $\Sigma$, in units of the critical surface mass density $\Sigma_{\mathrm{c}}$ :

$\kappa=\frac{\Sigma}{\Sigma_{\mathrm{c}}}, \quad \Sigma_{\mathrm{c}}=\frac{c^{2}}{4 \pi G} \frac{D_{\mathrm{s}}}{D_{d} D_{d s}}$,

where $D$ stands for angular diameter distance and the subscripts $s$, $d, d s$ indicate the distance from the observer to the source, from the observer to the lens, and from the lens to the source, respectively. In particular, Melchior et al. (2015) used $r_{1}=30$ arcmin and $r_{2}=45$ arcmin. This explains our choice of estimating the stellar mass surface density background in the range 30 to 50 arcmin, where its profile is also essentially flat. In Fig. 8 we also present the NFW mass profile derived by using the best-fitting parameters as found in Melchior et al. (2015) for this cluster. Given the similarity between the WL and stellar mass profiles, we try to fit the stellar mass one with an NFW projected mass profile, as the one derived in e.g. Oaxaca Wright \& Brainerd (2000):

$$
M(<x)=\left\{\begin{array}{rr}
\frac{3 \delta_{\mathrm{c}} M_{200 c}}{200 c_{200}^{3}}\left[\frac{2}{\sqrt{1-x^{2}}} \operatorname{arctanh} \sqrt{\frac{1-x}{1+x}}+\ln \left(\frac{x}{2}\right)\right] & (\text { if } x<1) \\
\frac{3 \delta_{\mathrm{c}} M_{200 \mathrm{c}}}{200 c_{200}^{3}}\left[1+\ln \left(\frac{1}{2}\right)\right] & \text { (if } x=1) \\
\frac{3 \delta_{\mathrm{c}} M_{200 \mathrm{c}}}{200 c_{200}^{3}}\left[\frac{2}{\sqrt{x^{2}-1}} \arctan \sqrt{\frac{x-1}{1+x}}+\ln \left(\frac{x}{2}\right)\right] & \text { (if } x>1)
\end{array}\right.
$$

where $x=R / r_{\mathrm{s}}, c_{200}=r_{200 \mathrm{c}} / r_{\mathrm{s}}$ is the concentration parameter and

$\delta_{\mathrm{c}}=\frac{200}{3} \frac{c_{200}^{3}}{\ln \left(1+c_{200}\right)-c_{200} /\left(1+c_{200}\right)}$.

Our non-linear least-squares fit uses the Levenberg-Marquardt algorithm and gives the following parameters for DES stellar mass profile: $M_{200 \mathrm{c}}^{\star}=(5.38 \pm 0.11) \times 10^{12} \mathrm{M}_{\odot}, c_{200}^{\star}=2.4 \pm 0.13$ with a reduced $\chi^{2}=0.6$.

From the DES stellar mass profile and the aperture mass densitometry total matter profile, we derive the stellar mass fraction $f_{\star}(<R)=M_{\star}(<R) / M_{\text {tot }}(<R)$, which is represented by the purple points in Fig. 9 . Within $r_{200 \mathrm{c}}$ radius, we find $f_{\star}^{\mathrm{DES}}\left(<r_{200 \mathrm{c}}\right)=$ $(6.8 \pm 1.7) \times 10^{-3}$, compatible within $1 \sigma$ in the outer regions with the result from Bahcall \& Kulier (2014): $f_{\star} \simeq(1.0 \pm 0.4) \times 10^{-2}$ above $\sim 300 h^{-1} \mathrm{kpc}$. In their paper, Bahcall \& Kulier (2014) examine the stellar fraction profile by stacking $>10^{5}$ SDSS groups and clusters, divided into three richness subsamples. ${ }^{8}$ Inside $r_{200 \mathrm{c}}$ we recover a lower stellar mass fraction compared to their work. The discrepancy can be explained in light of the different analyses carried out in Bahcall \& Kulier (2014):

(i) Bahcall \& Kulier (2014) stack clusters with different properties and at different redshifts.

\footnotetext{
${ }^{8}$ They define the richness $N_{200}$ as the number of galaxies in the red sequence with rest-frame $i$-band luminosity $L_{i}>0.4 L^{*}$ located within a radius $r_{200}^{\text {gals }}$ from the BCG (i.e. within the radius where the local galaxy overdensity is 200).
}

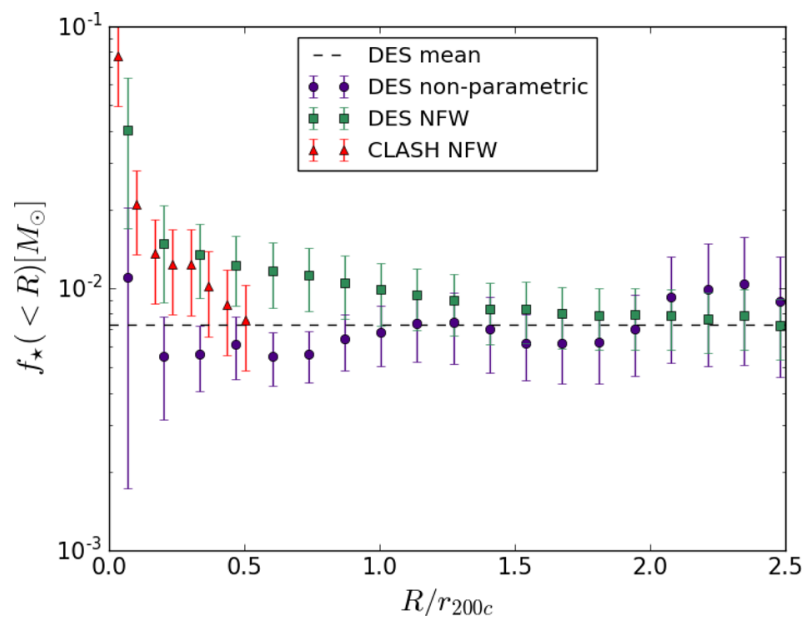

Figure 9. Cumulative radial distribution of the fraction of stellar mass of the galaxies in the cluster as computed in this work over the total mass from lensing studies. For the $\operatorname{DES} f_{\star}$, the total mass comes from the nonparametric reconstruction of the weak lensing shear profile done in Melchior et al. (2015) (purple points), or from their NFW best fit (green squares). For CLASH, $M_{\text {tot }}$ is a result of the Umetsu et al. (2016) NFW best fit for CLASH from a strong lensing, weak lensing and magnification joint analysis (red triangles). The mean DES stellar mass fraction from non-parametric weak lensing mass profile is $f_{\star}=(7.3 \pm 1.7) \times 10^{-3}$, and is represented by the dashed line. The radius $R$ is projected, and rescaled with $r_{200 \mathrm{c}}=2.2 \mathrm{Mpc}$. Error bars show the 68 per cent confidence level.

(ii) They included the contribution of the diffuse intracluster light (ICL), which increases $f_{\star}$ by a factor of 1.15 within $r_{200 c}$.

(iii) The luminosity profiles and weak lensing mass profiles have been de-projected to obtain 3D profiles in their work. On the other hand, considering the projected $f_{\star}$ means that we are including the contribution of the cluster outskirts along the line of sight when we look at cluster core. In these regions, the stellar mass fraction is lower, and this tends to reduce $2 \mathrm{D} f_{\star}$ at small radii with respect the $3 \mathrm{D}$ behaviour.

On the other hand, the average stellar mass of the Universe, estimated to be $f_{\star, \text { cosmic }}=(9 \pm 1) \times 10^{-3}$ (as derived in Bahcall \& Kulier 2014) is recovered outside $r_{200 c}$, as we would expect even for a projected profile.

Overall, no particular radial trend is found, in agreement with Bahcall \& Kulier (2014) and also with Andreon (2015), who studied the stellar-to-total mass ratio of three CLASH clusters at $z \sim 0.45$. Nevertheless, a radially varying profile might be hidden by the large errors. In order to reduce the latter, dominated by the weak lensing reconstructed mass, and have a precise estimation of the stellar mass fraction, we will need to apply the same reasoning to a large sample of DES clusters.

If we take the NFW mass profile with the lensing best-fitting parameters as total mass in $f_{\star}$, we get the green points in Fig. 9 for DES, and the red ones for CLASH. Towards the centre of the cluster these profiles are higher than the one previously discussed. This is due to the fact that in this case $M_{\text {tot }}$, as can be seen in equation (6), goes to zero for $R \rightarrow 0$, while the BCG stellar mass contributes to $M_{\star}$ up to very small radii. Moreover, the halo/cusp problem (see e.g. de Blok 2010) is a known problem of the NFW profile, which will therefore produce different results from a nonparametric mass profile from weak lensing. Use of the same dark matter halo parametrization brings the two data sets into agreement at the $1 \sigma$ level. 


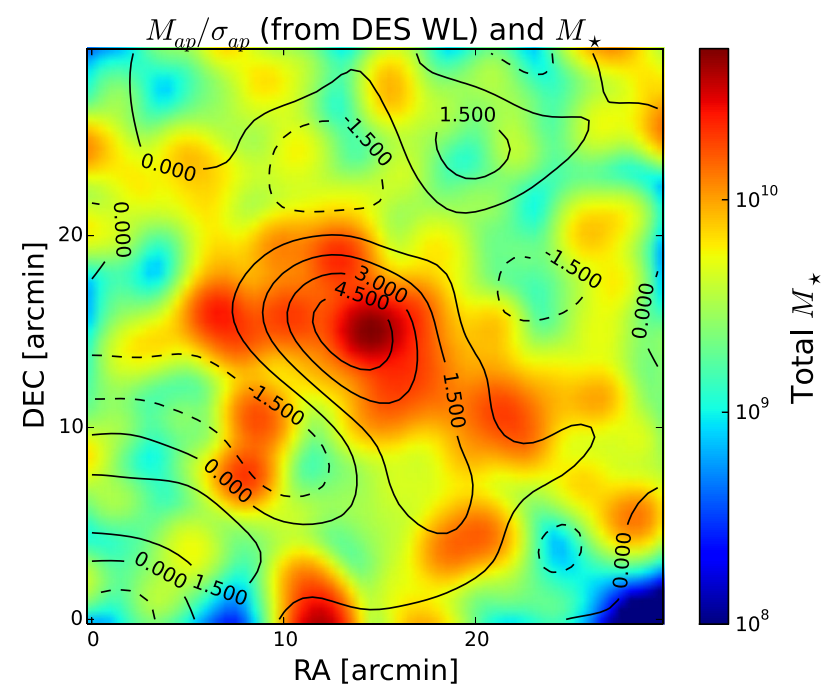

Figure 10. DES total stellar mass distribution (coloured density plot) compared to the mass map (i.e. map of $M_{\mathrm{ap}} / \sigma_{\mathrm{ap}}$, in contours) from the weak lensing analysis by Melchior et al. (2015). Both maps have a resolution of $0.4997 \mathrm{arcmin} / \mathrm{pixel}$ and have been smoothed with a Gaussian of $\sigma=1$ arcmin.

\subsection{DES stellar masses and weak lensing mass maps}

In this section, we explore the correlation between the stellar mass maps and the DES weak lensing mass map by Melchior et al. (2015). Melchior et al. (2015) adopted the aperture-mass technique from Schneider (1996) and computed the map of $M_{\mathrm{ap}} / \sigma_{\text {ap }}$ for this cluster, where $M_{\text {ap }}$ is the aperture mass and it is defined as the sum of all ellipticity measurements $\epsilon_{t}\left(\vartheta_{j}\right)$ inside a circular aperture:

$M_{\mathrm{ap}}(\vartheta)=\sum_{j} Q\left(\left|\vartheta-\vartheta_{j}\right|\right) \epsilon_{t}\left(\vartheta_{j}\right)$.

In equation (8) $Q$ is a weight function, and the ellipticities are computed with respect to the centre $\vartheta$ of the circular aperture. The variance of the aperture mass is given by:

$\sigma_{M_{\mathrm{ap}}}^{2}=\frac{\sigma_{\epsilon}^{2}}{2} \sum_{j} Q^{2}\left(\left|\vartheta-\vartheta_{j}\right|\right)$.

In Fig. 10 we show the DES aperture mass map (black contours) and our stellar mass map (coloured density map) in $30 \times 30 \mathrm{arcmin}^{2}$ around the BCG position. Both maps have a resolution of 0.4997 arcmin/pixel and have been smoothed with a Gaussian of $\sigma=1$ arcmin.

An elongated structure spanning for $\sim 4 \mathrm{Mpc}$ around the BCG is clearly present in both maps, as well as a few clumps lying inside and outside the $r_{200 \mathrm{c}}$ radius. Note that the mass structures that can be seen in the total mass map may lie outside the cluster but still cause the lensing, as they are along the line of sight, while the DES galaxies considered here are only those at the cluster redshift. This fact partially explains why the peaks and minima in the stellar mass and aperture mass maps may be not always coincident. Also, the peak of the stellar mass distribution coincides with the BCG position, while the weak lensing map shows a small offset of the peak from the BCG: Gruen et al. (2013) already addressed this effect to the expected shape noise studied by Dietrich et al. (2012).

The correlation that can be seen by eye between $M_{\text {ap }} / \sigma_{\text {ap }}$ and $\log M_{\star}$ is quantified by a Pearson coefficient of $r=0.30$ when crosscorrelating the maps pixel by pixel. Again, the correlation expected between stellar mass and total matter (quantified in this case by the aperture mass and convergence) in a cluster is diminished by the fact that the gravitational lensing gives integrated information about the mass along the line of sight.

\section{CONCLUSIONS}

We compared the catalogues derived from DES and CLASH observations of the galaxy cluster RXC J2248.7-4431, treating CLASH as a validation set for DES. Photometric redshifts and stellar masses for both data sets were computed using LEPHARE, and we found that stellar masses can be estimated with good precision with DES, despite the lower number of bands available. Gravitational lensing results from both DES and CLASH were used to compare stellar and total mass maps, as well as the mass profiles and stellar mass fraction.

Following are the conclusions.

(i) HST data can be used as a validation set for DES data and results. We expect three more CLASH clusters (Abell 383, MACS0416.1-2403 and MACS0429.6-0253) to be included in the whole DES footprint. We found that in this case using the CLASS_STAR parameters with the mentioned cut is more efficient than adopting the SPREAD_MODEL_I with the cut at 0.003 .

(ii) DES photo-zs are compatible with the 17 HST filters photo$z$ s within the DES requirements. The $z \sim 0.1$ offset observed in the DES photo- $z$ s is due to a colour-redshift degeneracy that cannot be broken without UV bands at redshifts below 0.4 . We found that such offset would percolate into the stellar mass estimates and bias the results by $\sim 0.16$ dex. In order to perform stellar mass studies, we therefore overcame the problem of the redshift estimation for single cluster members by devising a technique as follows. This method separately treats the red galaxies, as found by REDMAPPER, and the blue galaxies. The redshift information can either be the spectroscopic redshift of the cluster, if available, or the REDMAPPER cluster photometric redshift (as REDMAPPER photo- $z$ s are estimated with high precision). In order to estimate the blue galaxies' contribution to the stellar mass, we perform a background subtraction which is only possible thanks to the DECam wide field of view.

(iii) We then estimated total stellar mass and stellar mass fraction profiles for both DES and CLASH, reaching large radii with DES. Within the projected $r_{200 c}$ radius, we find a fraction of stellar mass over total mass (derived from weak lensing) $f_{\star}\left(<r_{200 \mathrm{c}}\right)=(6.8 \pm 1.7) \times 10^{-3}$ with DES, which is compatible with other recent measurements from an independent data set (Bahcall \& Kulier 2014).

(iv) On cosmological scales, the ratio of baryon to total matter densities is $\Omega_{\mathrm{b}} / \Omega_{m} \approx 16$ percent (e.g. Planck Collaboration XIII et al. 2015). In the cluster core we find that the ratio of stellar mass to total matter is $\sim 0.7$ per cent. This means that if the cluster distribution is representative, then only 4 per cent of the baryons are locked into stars (compatible within $2 \sigma$ with Fukugita \& Peebles 2004).

(v) The stellar mass fraction profiles we derive from DES and CLASH are compatible within $1 \sigma$, provided that the same parametrization is used for the total matter halo profile.

(vi) HST clusters can be used to test and calibrate stellar mass estimates. In future works we plan to test the stellar mass-to-light ratio derived from DES to that from $H S T$, as this should be nominally invariant under changes in galaxy apertures (that are different between the two data sets used in this work), and therefore better matched than $M_{\star}$ between the surveys. In addition, an extensive spectroscopic campaign carried out with the Very Large Telescope 
(VLT) (CLASH-VLT, Rosati et al. 2014) is currently providing thousands of spectra for 14 CLASH clusters. In the future, we plan to use this survey to further test our technique: this data set will include spectra up to very large cluster radii, making CLASH-VLT ideal to test stellar masses on the large scales explored by DES. CLASH-VLT analysis of stellar masses for the cluster RXJ2248 is in course of preparation (Annunziatella et al., in preparation), and stellar mass density profiles have been studied recently for other clusters (Annunziatella et al. 2014, 2016).

(vii) In the future, we plan to apply the same techniques to a sample of 100000 stacked DES clusters and deduce important information about galaxy evolution by looking at the relation between stellar and total mass, as a function of radii and redshift, at the Stellar Mass Function and at the stellar mass environment dependence. The stacking of the whole DES sample would allow us to estimate $f_{\star}$ with an error significantly smaller than that given here.

\section{ACKNOWLEDGEMENTS}

AP acknowledges the UCL PhD studentship. OL acknowledges support from a European Research Council Advanced Grant FP7/291329, which also supported M. Banerji and S. Jouvel.

DG and SS were supported by SFB-Transregio 33 'The Dark Universe' by the Deutsche Forschungsgemeinschaft (DFG) and the DFG cluster of excellence 'Origin and Structure of the Universe'. DG was also supported by NASA through the Einstein Fellowship Program, grant PF5-160138.

TJ acknowledges support from the DOE grant DE-SC0013541. This work has benefitted by data taken by the CLASH collaboration.

A. P and OL acknowledge N. Bahcall and M. Milgrom for stimulating discussions about this work.

Funding for the DES Projects has been provided by the U.S. Department of Energy, the U.S. National Science Foundation, the Ministry of Science and Education of Spain, the Science and Technology Facilities Council of the United Kingdom, the Higher Education Funding Council for England, the National Center for Supercomputing Applications at the University of Illinois at Urbana-Champaign, the Kavli Institute of Cosmological Physics at the University of Chicago, the Center for Cosmology and Astro-Particle Physics at the Ohio State University, the Mitchell Institute for Fundamental Physics and Astronomy at Texas A\&M University, Financiadora de Estudos e Projetos, Fundação Carlos Chagas Filho de Amparo à Pesquisa do Estado do Rio de Janeiro, Conselho Nacional de Desenvolvimento Científico e Tecnológico and the Ministério da Ciência, Tecnologia e Inovação, the Deutsche Forschungsgemeinschaft and the Collaborating Institutions in the Dark Energy Survey.

The Collaborating Institutions are Argonne National Laboratory, the University of California at Santa Cruz, the University of Cambridge, Centro de Investigaciones Energéticas, Medioambientales y Tecnológicas-Madrid, the University of Chicago, University College London, the DES-Brazil Consortium, the University of Edinburgh, the Eidgenössische Technische Hochschule (ETH) Zürich, Fermi National Accelerator Laboratory, the University of Illinois at Urbana-Champaign, the Institut de Ciències de l'Espai (IEEC/CSIC), the Institut de Física d'Altes Energies, Lawrence Berkeley National Laboratory, the Ludwig-Maximilians Universität München and the associated Excellence Cluster Universe, the University of Michigan, the National Optical Astronomy Observatory, the University of Nottingham, The Ohio State University, the University of Pennsylvania, the University of Portsmouth, SLAC
National Accelerator Laboratory, Stanford University, the University of Sussex, and Texas A\&M University.

The DES data management system is supported by the National Science Foundation under Grant Number AST-1138766. The DES participants from Spanish institutions are partially supported by MINECO under grants AYA2012-39559, ESP201348274, FPA2013-47986, and Centro de Excelencia Severo Ochoa SEV-2012-0234. Research leading to these results has received funding from the European Research Council under the European Unions Seventh Framework Programme (FP7/2007-2013) including ERC grant agreements 240672, 291329, and 306478.

\section{REFERENCES}

Abell G. O., Corwin H. G., Jr., Olowin R. P., 1989, ApJS, 70, 1

Acquaviva V., Raichoor A., Gawiser E., 2015, ApJ, 804, 8

Andreon S., 2015, A\&A, 575, A108

Annunziatella M. et al., 2014, A\&A, 571, A80

Annunziatella M. et al., 2016, A\&A, 585, A160

Arnouts S., Cristiani S., Moscardini L., Matarrese S., Lucchin F., Fontana A., Giallongo E., 1999, MNRAS, 310, 540

Bahcall N. A., Kulier A., 2014, MNRAS, 439, 2505

Banerji M. et al., 2013, MNRAS, 431, 2209

Benítez N. et al., 2004, ApJS, 150, 1

Bertin E., Arnouts S., 1996, Astron. Astrophys. Suppl., 117, 393

Böhringer H. et al., 2004, A\&A, 425, 367

Bonnett C. et al., 2015, preprint (arXiv:astro-ph/1507.05909)

Bouy H., Bertin E., Moraux E., Cuillandre J.-C., Bouvier J., Barrado D., Solano E., Bayo A., 2013, A\&A, 554, A101

Bruzual G., Charlot S., 2003, MNRAS, 344, 1000

Calzetti D., Armus L., Bohlin R. C., Kinney A. L., Koornneef J., StorchiBergmann T., 2000, ApJ, 533, 682

Chabrier G., 2003, PASP, 115, 763

Clowe D., Luppino G. A., Kaiser N., Henry J. P., Gioia I. M., 1998, ApJ, 497, L61

Conroy C., 2013, ARA\&A, 51, 393

Crocce M. et al., 2016, MNRAS, 455, 4301

de Blok W. J. G., 2010, Adv. Astron., 2010, 789293

Desai S. et al., 2012, ApJ, 757, 83

Diehl H. T. et al., 2014, in SPIE Conf. Ser. Vol. 9149, Society of PhotoOptical Instrumentation Engineers (SPIE) Conference Series, p. 91490V

Dietrich J. P., Böhnert A., Lombardi M., Hilbert S., Hartlap J., 2012, MNRAS, 419, 3547

Eisenstein D. J. et al., 2001, AJ, 122, 2267

Flaugher B. et al., 2015, AJ, 150, 150

Fukugita M., Peebles P. J. E., 2004, ApJ, 616, 643

Gómez P. L. et al., 2012, AJ, 144, 79

Górski K. M., Hivon E., Banday A. J., Wandelt B. D., Hansen F. K., Reinecke M., Bartelmann M., 2005, ApJ, 622, 759

Gruen D. et al., 2013, MNRAS, 432, 2

Ilbert O. et al., 2006, A\&A, 457, 841

Ilbert O. et al., 2009, ApJ, 690, 1236

Ilbert O. et al., 2010, ApJ, 709, 644

Jouvel S. et al., 2014, A\&A, 562, A86

Koekemoer A. M. et al., 2011, ApJS, 197, 36

Maughan B. J., Jones C., Forman W., Van Speybroeck L., 2008, ApJS, 174, 117

Melchior P. et al., 2015, MNRAS, 449, 2219

Mohr J. J. et al., 2012, Proc. SPIE, 8451, 84510D

Monna A. et al., 2014, MNRAS, 438, 1417

Navarro J. F., Frenk C. S., White S. D. M., 1996, ApJ, 462, 563

Oaxaca Wright C., Brainerd T. G., 2000, ApJ, 534, 34

Planck Collaboration XIII et al., 2015, preprint (arXiv:astro-ph/1502.01589)

Postman M. et al., 2012, ApJS, 199, 25

Rosati P. et al., 2014, The Messenger, 158, 48 
Rozo E., Rykoff E. S., Becker M., Reddick R. M., Wechsler R. H., 2015, MNRAS, 453, 38

Rykoff E. S. et al., 2014, ApJ, 785, 104

Rykoff E. S. et al., 2016, ApJS, 224, 1

Sánchez C. et al., 2014, MNRAS, 445, 1482

Schneider P., 1996, MNRAS, 283, 837

Sevilla I. et al., 2011, preprint (arXiv:astro-ph/1109.6741)

Soumagnac M. T. et al., 2015, MNRAS, 450, 666

Suchyta E. et al., 2016, MNRAS, 457, 786

Swanson M. E. C., Tegmark M., Hamilton A. J. S., Hill J. C., 2008, MNRAS, 387,1391

The Dark Energy Survey Collaboration 2005, preprint (arXiv:astro-ph/0510346)

Umetsu K., Zitrin A., Gruen D., Merten J., Donahue M., Postman M., 2016, ApJ, 821, 116

Zhang Y., McKay T. A., Bertin E., Jeltema T., Miller C. J., Rykoff E., Song J., 2015, PASP, 127, 1183

\section{APPENDIX A: MAGNITUDE COMPARISON}

Considering only those matched sources with a $S / N>10$ in the DES $i$ band, excluding stars and objects with SExTRACTOR FLAGS $>3$ (in order to exclude objects with saturated pixels or corrupted data, but include objects that were initially blended) we are left with 327 sources observed in the $g$ and $r$ bands, and 331 in the $i$ and $z$ bands. The differences $\Delta_{\mathrm{m}}=m_{\mathrm{DES}}-m_{\mathrm{CLASH}}$ are plotted in Fig. A1, as well as the DES magnitudes as a function of the CLASH ones for the matched sources. The magnitudes plotted are SEXTRACTOR isophotal magnitudes MAG_ISO for both DES and CLASH. $\Delta_{\mathrm{m}}$ in the griz bands has been corrected for the magnitude shifts due to the differences between these DES and HST filters. The offsets have been computed using two 'extreme case' SED templates (one elliptical, one irregular) at the cluster redshift. We have not taken into consideration the $Y$-band offset as the HST and DES filters are too different, but we still report the comparison for completeness.

Fig. A1 shows an offset in the DES magnitudes, especially in the gri bands, which may be due to different choices of threshold or background when running SExTRACTOR. Nevertheless, the linear trend is clear, bringing to Pearson coefficients between 0.91 and 0.98 in all bands. The higher scatter that we could expect in the $Y$ band (as the corresponding CLASH filter is the F105W, which is much more spread towards the infrared than the DES $Y$ filter) is actually compensated for by higher DES photometric errors. The mean difference in magnitude $\Delta_{\mathrm{m}}$ between the two data sets is 0.13 , $0.04,-0.07,-0.07$ and 0.08 in the griz $Y$ bands, respectively.

\section{APPENDIX B: PHOTO- $z$ CONSISTENCY TEST}

In this section we test whether the fact that we assumed a photo- $z$ that was computed with a certain set of templates to then compute the stellar mass with a larger set of templates is consistent. With photometric surveys data, it is a common method to perform estimation of the photometric redshift and SED fitting in two steps, which involve fixing the redshift of a galaxy at the best-fitting value obtained in the first step. Although this may not be the most elegant way of solving the problem of the lack of spectroscopic information, it has been proven to lead to only a small bias in the SED fitting
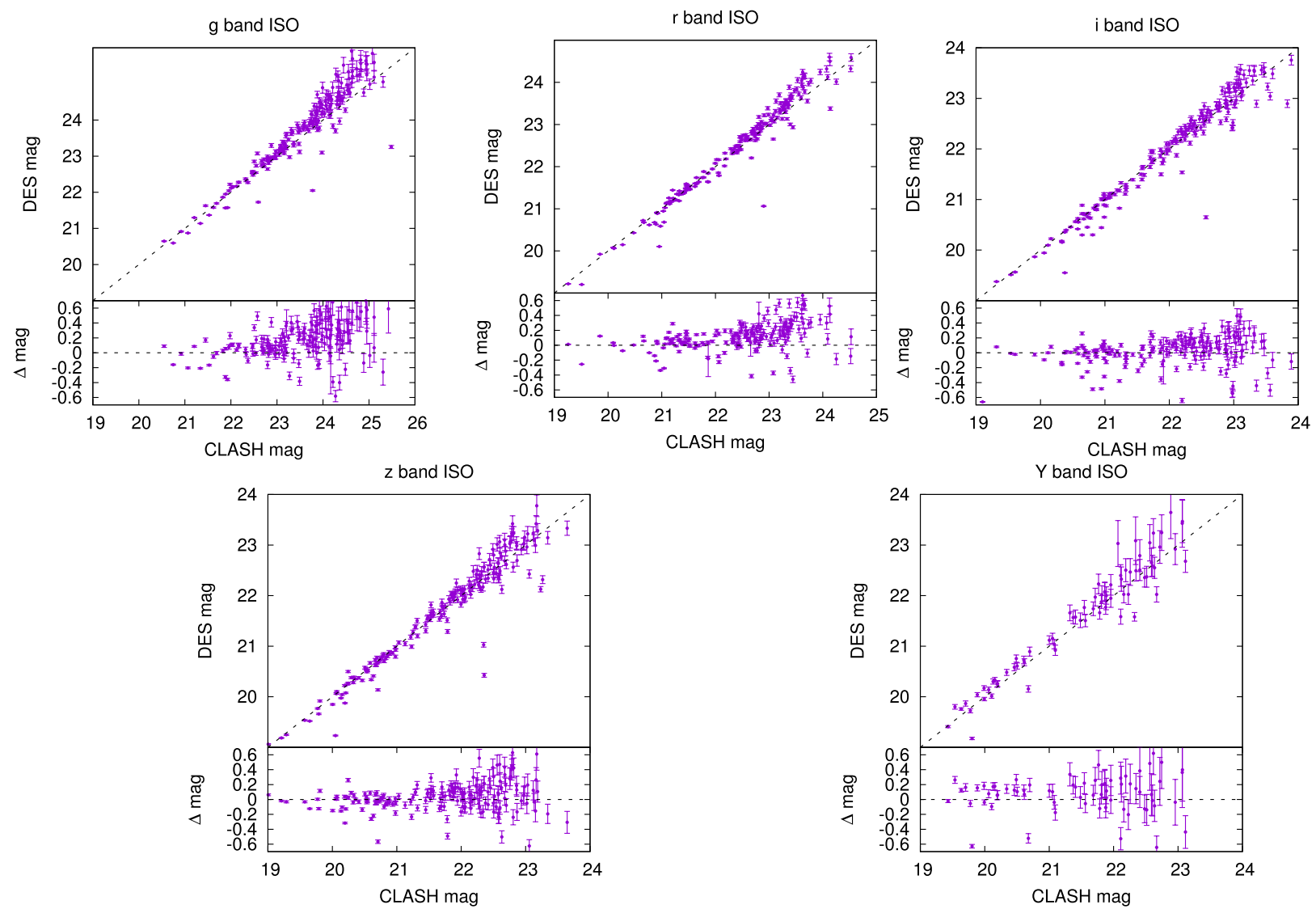

Figure A1. DES magnitudes compared to CLASH magnitudes, with bottom plots of $\Delta_{\mathrm{m}}=m_{\mathrm{CLASH}}-m_{\mathrm{DES}}$ in the $g, r, i$ and $z$ bands for the matched sources that satisfy $\mathrm{S} / \mathrm{N}>10$ and filtering the sources with FLAGS $>3$. CLASH error bars are not plotted for visualization purposes, while those on the DES magnitudes represent the $1 \sigma$ error. The dashed black lines represent the ideal case $m_{\mathrm{CLASH}}=m_{\mathrm{DES}}$. 

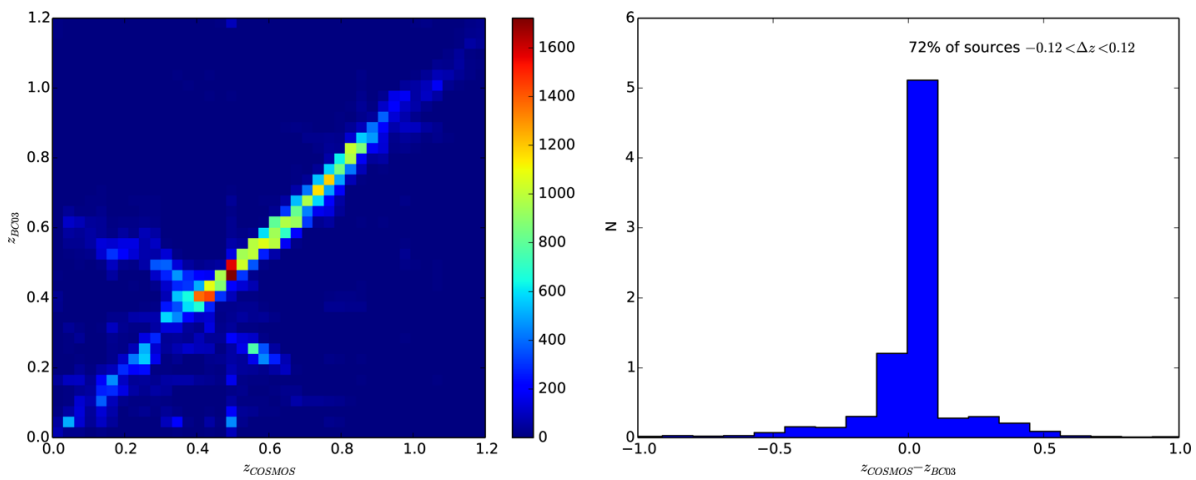

Figure B1. Left panel: comparison of the photo-zs computed using the Bruzual and Charlot (2003) templates and those using the COSMOS templates. Right panel: residuals of the photo- $z$ s computed using the two different set of templates.

parameters, when compared to results given by the simultaneous estimation of redshift and stellar mass (see Acquaviva et al. 2015). Moreover, here we can take advantage of the prior information that this is a cluster.

In order to test the consistency of the photo- $z$ choice, we want to show that the resulting photo- $z$ is not drastically dependent on the choice of templates. Therefore, we compare the photo- $z$ s given by Le Phare when using the COSMOS templates and the $\mathrm{BC} 03$ ones. A comparison is shown in Fig. B1 for all the galaxies in the DES field of view. We retrieve that 71 per cent of the galaxies have $|\Delta z|=\left|z_{\mathrm{BC} 03}-z_{\mathrm{COSMOS}}\right|<0.12$.

${ }^{1}$ Department of Physics and Astronomy, University College London, Gower Street, London WC1E 6BT, UK

${ }^{2}$ Institute of Astronomy, University of Cambidge, Madingley Road, Cambridge CB3 OHA, UK

${ }^{3}$ SLAC National Accelerator Laboratory, Menlo Park, CA 94025, USA

${ }^{4}$ Kavli Institute for Particle Astrophysics \& Cosmology, P. O. Box 2450, Stanford University, Stanford, CA 94305, USA

${ }^{5}$ University Observatory Munich, Scheinerstrasse 1, D-81679 Munich, Germany

${ }^{6}$ Max Planck Institute for Extraterrestrial Physics, Giessenbachstrasse, D-85748 Garching, Germany

${ }^{7}$ Department of Astrophysical Sciences, Princeton University, Princeton, $N J$ 08544, USA

${ }^{8}$ Institut de Fisica d'Altes Energies (IFAE), The Barcelona Institute of Science and Technology, Campus UAB, E-08193 Bellaterra (Barcelona), Spain

${ }^{9}$ Fermi National Accelerator Laboratory, Batavia, IL 60510, USA

${ }^{10}$ Department of Physics, University of California, Santa Cruz, CA 95064 , USA

${ }^{11}$ Department of Physics and Astronomy, Pevensey Building, University of Sussex, Brighton BN1 9QH, UK

${ }^{12}$ Department of Physics, University of Arizona, Tucson, AZ 85721, USA

${ }^{13}$ Department of Physics and Astronomy, University of Pennsylvania, Philadelphia, PA 19104, USA

${ }^{14}$ Physics Department, University of Michigan, 450 Church Street, Ann Arbor, MI 48109, USA

${ }^{15}$ Cerro Tololo Inter-American Observatory, National Optical Astronomy Observatory, Casilla 603, La Serena, Chile

${ }^{16}$ Department of Physics and Electronics, Rhodes University, PO Box 94, Grahamstown, 6140, South Africa

${ }^{17}$ CNRS, UMR 7095, Institut d'Astrophysique de Paris, F-75014, Paris, France

${ }^{18}$ Sorbonne Universités, UPMC Univ Paris 06, UMR 7095, Institut d'Astrophysique de Paris, F-75014, Paris, France

${ }^{19}$ Institute of Cosmology \& Gravitation, University of Portsmouth, Portsmouth PO1 3FX, UK
${ }^{20}$ Laboratório Interinstitucional de e-Astronomia - LIneA, Rua Gal. José Cristino 77, Rio de Janeiro, RJ - 20921-400, Brazil

${ }^{21}$ Observatório Nacional, Rua Gal. José Cristino 77, Rio de Janeiro, RJ 20921-400, Brazil

${ }^{22}$ Department of Astronomy, University of Illinois, 1002 W. Green Street, Urbana, IL 61801, USA

${ }^{23}$ National Center for Supercomputing Applications, 1205 West Clark St., Urbana, IL 61801, USA

${ }^{24}$ Institut de Ciències de l'Espai, IEEC-CSIC, Campus UAB, Carrer de Can Magrans, s/n, E-08193 Bellaterra, Barcelona, Spain

${ }^{25}$ School of Physics and Astronomy, University of Southampton, Southampton SO17 1BJ, UK

${ }^{26}$ Excellence Cluster Universe, Boltzmannstr. 2, D-85748 Garching, Germany

${ }^{27}$ Faculty of Physics, Ludwig-Maximilians University, Scheinerstr. 1, D-81679 Munich, Germany

${ }^{28}$ Department of Astronomy, University of Michigan, Ann Arbor, MI 48109, USA

${ }^{29}$ Kavli Institute for Cosmological Physics, University of Chicago, Chicago, IL 60637, USA

${ }^{30}$ Department of Astronomy, University of California, Berkeley, 501 Campbell Hall, Berkeley, CA 94720, USA

${ }^{31}$ Lawrence Berkeley National Laboratory, 1 Cyclotron Road, Berkeley, CA 94720, USA

${ }^{32}$ Center for Cosmology and Astro-Particle Physics, The Ohio State University, Columbus, OH 43210, USA

${ }^{33}$ Department of Physics, The Ohio State University, Columbus, OH 43210 , USA

${ }^{34}$ Australian Astronomical Observatory, North Ryde, NSW 2113, Australia

${ }^{35}$ George P. and Cynthia Woods Mitchell Institute for Fundamental Physics and Astronomy, and Department of Physics and Astronomy, Texas A\&M University, College Station, TX 77843, USA

${ }^{36}$ Departamento de Física Matemática, Instituto de Física, Universidade de São Paulo, CP 66318, CEP 05314-970, São Paulo, SP, Brazil

${ }^{37}$ Institució Catalana de Recerca i Estudis Avançats, E-08010 Barcelona, Spain

${ }^{38}$ Jet Propulsion Laboratory, California Institute of Technology, 4800 Oak Grove Dr., Pasadena, CA 91109, USA

${ }^{39}$ Centro de Investigaciones Energéticas, Medioambientales y Tecnológicas (CIEMAT), Madrid, Spain

${ }^{40}$ Argonne National Laboratory, 9700 South Cass Avenue, Lemont, IL 60439, USA

This paper has been typeset from a $\mathrm{T}_{\mathrm{E}} \mathrm{X} / \mathrm{L} \mathrm{T}_{\mathrm{E}} \mathrm{X}$ file prepared by the author. 